\title{
The ab initio model potential method: Lanthanide and actinide elements
}

\author{
Luis Seijo, ${ }^{\text {a) }}$ Zoila Barandiarán, and Eduardo Harguindey \\ Departamento de Química and Instituto Universitario de Ciencia de Materiales Nicolás Cabrera, \\ C-XIV Universidad Autónoma de Madrid, 28049 Madrid, Spain
}

(Received 2 August 2000; accepted 12 October 2000)

\begin{abstract}
In this paper we present relativistic core ab initio model potentials based on atomic Cowan-Griffin calculations, together with Wood-Boring spin-orbit operators and optimized Gaussian valence basis sets, for the lanthanide elements $\mathrm{Ce}$ to $\mathrm{Lu}$ and for the actinide elements Th to Lr. This completes the chemically relevant part of the Periodic Table. A $[\mathrm{Kr}, 4 d]$ core was chosen for $\mathrm{Ce}-\mathrm{Lu}$ and a $[\mathrm{Xe}, 4 f, 5 d]$ core was chosen for Th-Lr. Minimal $(14 s 10 p 9 d 8 f) /[2 s 1 p 1 d 1 f]$ and $(14 s 10 p 11 d 9 f) /[2 s 1 p 1 d 1 f]$ valence basis sets were, respectively, optimized for Ce-Lu and Th-Lr, and a $[6 s 5 p 5 d 4 f]$ contraction is recommended for all these 28 elements in molecular calculations. The atomic and molecular results show the same good quality already observed for the main-group elements and the transition metal elements. (C) 2001 American Institute of Physics.
\end{abstract}

[DOI: 10.1063/1.1330209]

\section{INTRODUCTION}

Effective core potential methods (ECP) are accepted as efficient tools to carry out spin-free and spin-orbit relativistic $a b$ initio calculations in molecules and crystals. They are especially indicated when heavy elements are involved.

Two families of relativistic ECP methods exist: those relying on the pseudo-orbital transformation, ${ }^{1}$ which produce nodeless valence pseudo-orbitals and are known as pseudopotential methods, and those based on the Huzinaga-Cantu equation, ${ }^{2}$ which lead to valence orbitals with the same nodal structure as the all-electron orbitals and are know as model potential methods. Within the latter kind, the $a b$ initio model potential method (AIMP) ${ }^{3}$ resulted from the implementation of two ideas: ${ }^{4}$ (i) the core model potentials are obtained directly from the frozen core orbitals, without resorting to parametrization procedures based on the valence orbitals, and (ii) the components of the core model potentials must mimic the operators that they substitute as much as possible, while reducing the computing time. Spin-free relativistic core AIMPs derived from atomic Cowan-Griffin calculations ${ }^{5}$ and extended to include spin-orbit coupling effects according to Wood and Boring suggestions ${ }^{6}$ constitute the so-called relativistic WB-AIMPs, ${ }^{7}$ which are used with optimized valence basis sets. In addition to the nonrelativistic ones, the ingredients of the WB-AIMP method have been produced, successfully monitored, and used for the main-group elements and for the three series of transition metal elements. ${ }^{4,7-11}$ The quality of the one-electron effective spinorbit Wood-Boring operators was shown to be very high in atomic and molecular calculations. ${ }^{12,13}$ Also, the expected good performance of this method for $f$ elements has been confirmed in relativistic spin-orbit calculations on $\mathrm{Ce}$ and spin-free calculations on $\mathrm{CeO}$ (Ref. 14) (Ce is recognized as a difficult case $\left.\mathrm{e}^{15,16}\right)$ and taken as an indication that one could

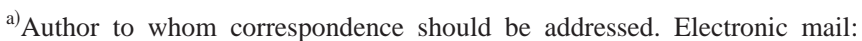
luis.seijo@uam.es
}

safely proceed with the lanthanide and actinide series.

In order to complete the chemically relevant part of the Periodic Table, we produced and present here the ingredients of the WB-AIMP method for the lanthanide elements Ce to $\mathrm{Lu}$ and for the actinide elements Th to Lr. (For these elements, several sets of relativistic pseudopotentials are available in the literature. ${ }^{16-20}$ ) We present $[\mathrm{Kr}, 4 d]$ core AIMPs together with $5 s, 5 p, 4 f, 5 d, 6 s$ optimized valence basis sets and Wood-Boring spin-orbit operators for $\mathrm{Ce}-\mathrm{Lu}$, and $[\mathrm{Xe}, 4 f, 5 d]$ core AIMPs with $6 s, 6 p, 5 f, 6 d, 7 s$ optimized valence basis sets and Wood-Boring spin-orbit operators for Th-Lr. It is worth noticing that the Gaussian primitive functions that have been optimized here with the Cowan-Griffin relativistic Hamiltonian are expected to be transferable to relativistic AIMPs based on the Douglas-Kroll-Hess Hamiltonian, ${ }^{21,22}$ as was the case of the transition metal elements from $\mathrm{Sc}$ to $\mathrm{Hg}^{23,24}$

\section{II. $A B$ INITIO MODEL POTENTIALS AND VALENCE BASIS SETS}

We summarize here the procedure leading to (1) the spin-free relativistic core $a b$ initio model potentials derived from Cowan-Griffin atomic calculations (CG-AIMP); (2) the Wood-Boring one-electron spin-orbit operators which are added to the CG-AIMP Hamiltonian in order to produce the spin-dependent WB-AIMP Hamiltonian; and (3) the valence basis sets. The details have been fully described in Refs. 3, 4, and 7. All these data are available from the authors $^{25}$ and are presented in the Supplementary Material Section of Ref. 26 as E-PAPS document files.

\section{A. WB-AIMP Hamiltonian}

The spin-dependent relativistic WB-AIMP Hamiltonian of a molecule, $\hat{H}^{\text {WB-AIMP }}$, is the sum of a spin-free relativistic Hamiltonian, $\hat{H}^{\text {CG-AIMP }}$, and a pure spin-orbit Hamiltonian, $\hat{H}^{\mathrm{SO}}$ 
TABLE I. Core/valence partitions and valence basis sets used for the lanthanide and actinide elements.

\begin{tabular}{lllll}
\hline \hline & \multicolumn{1}{c}{ Label } & \multicolumn{1}{c}{ Core orbitals } & Valence orbitals & \multicolumn{1}{c}{ Minimal valence basis set } \\
\hline $\mathrm{Ce}-\mathrm{Lu}$ & {$[\mathrm{Kr}, 4 d]$} & $\mathrm{K}, \mathrm{L}, \mathrm{M}, 4 s, 4 p, 4 d$ & $5 s, 5 p, 4 f, 5 d, 6 s$ & $(14 s 10 p 9 d 8 f) /[2 s 1 p 1 d 1 f]$ \\
$\mathrm{Th}-\mathrm{Lr}$ & {$[\mathrm{Xe}, 4 f, 5 d]$} & $\mathrm{K}, \mathrm{L}, \mathrm{M}, \mathrm{N}, 5 s, 5 p, 5 d$ & $6 s, 6 p, 5 f, 6 d, 7 s$ & $(14 s 10 p 11 d 9 f) /[2 s 1 p 1 d 1 f]$ \\
\hline
\end{tabular}

$$
\hat{H}^{\mathrm{WB}-\mathrm{AIMP}}=\hat{H}^{\mathrm{CG}-\mathrm{AIMP}}+\hat{H}^{\mathrm{SO}} .
$$

For a molecule with $N_{\text {val }}$ valence electrons and $N_{\text {nuc }}$ nuclei (each with nuclear charge $Z^{I}$ and number of core electrons $\left.Z_{\text {core }}^{I}\right)$, the spin-free Hamiltonian reads

$$
\begin{aligned}
\hat{H}^{\mathrm{CG}-\mathrm{AIMP}}= & \sum_{i}^{N_{\text {val }}} \hat{h}^{\mathrm{CG}-\mathrm{AIMP}}(i)+\sum_{i<j}^{N_{\text {val }}} \frac{1}{r_{i j}} \\
& +\sum_{I<J}^{N_{\text {nuc }}} \frac{\left(Z^{l}-Z_{\text {core }}^{I}\right)\left(Z^{J}-Z_{\text {core }}^{J}\right)}{R_{I J}},
\end{aligned}
$$

with $\hat{h}^{\text {CG-AIMP }}(i)$, the one-electron spin-free relativistic Cowan-Griffin $a b$ initio model potential, defined by

$$
\begin{aligned}
\hat{h}^{\mathrm{CG}-\operatorname{AIMP}}(i)= & -\frac{1}{2} \hat{\nabla}_{i}^{2}+\sum_{I}^{N_{\text {nuc }}}\left[-\frac{Z^{I}-Z_{\text {core }}^{I}}{r_{i}}\right. \\
& +\sum_{k} \frac{C_{k}^{I} \exp \left(-\alpha_{k}^{I} r_{i}^{2}\right)}{r_{i}} \\
& +\sum_{l} \sum_{m=-l}^{+l} \sum_{a, b}\left|\chi_{a l m}^{I}\right\rangle A_{l ; a b}^{I, M P}\left\langle\chi_{b l m}^{I}\right| \\
& \left.+\sum_{c \in \text { core }}\left(-2 \varepsilon_{c}^{I}\right)\left|\phi_{c}^{I}\right\rangle\left\langle\phi_{c}^{I}\right|\right] .
\end{aligned}
$$

This operator is a practical, approximate representation of the corresponding frozen-core operator ${ }^{27}$

$$
\begin{aligned}
\hat{h}^{\mathrm{CG}-\mathrm{FC}}(i)= & -\frac{1}{2} \hat{\nabla}_{i}^{2}+\sum_{I}^{N_{\text {nuc }}}\left[-\frac{Z^{I}-Z_{\text {core }}^{I}}{r_{i}}-\frac{Z_{\text {core }}^{I}}{r_{i}}\right. \\
& +2 \sum_{c \in \text { core }} \hat{J}_{c}^{I}\left(r_{i}\right)-\sum_{c \in \text { core }} \hat{K}_{c}^{I}+\hat{V}_{M V}^{I} \\
& \left.+\hat{V}_{D}^{I}+\sum_{c \in \text { core }}\left(-2 \varepsilon_{c}^{I}\right)\left|\phi_{c}^{I}\right\rangle\left\langle\phi_{c}^{I}\right|\right],
\end{aligned}
$$

which is made of the nonrelativistic kinetic energy operator and a sum of atomic operators that includes, for each of them, the nuclear attraction, the core Coulomb and exchange operators, the Cowan-Griffin-Wood-Boring valence massvelocity and Darwin operators, and a final term resulting from the linear-independency conditions between core and valence orbitals. ${ }^{2}$ In Eq. (4), $\hat{V}_{M V}^{I}+\hat{V}_{D}^{I}$ reads

$$
\hat{V}_{M V}^{I}+\hat{V}_{D}^{I}=\sum_{n l \in \text { valence }} \hat{O}_{l}^{I}\left(V_{M V, n l}^{I}+V_{D, n l}^{I}\right) \hat{O}_{l}^{I},
$$

where $V_{M V, n l}^{I}+V_{D, n l}^{I}$ are the orbital-dependent mass-velocity plus Darwin potentials of Cowan-Griffin-Wood-Woring (related to but different from Pauli's mass-velocity and Darwin operators $)^{5,6}$ and the angular projectors made of spherical harmonics

$$
\hat{O}_{l}^{I}=\sum_{m=-l}^{+l}\left|Y_{l m}^{I}\right\rangle\left\langle Y_{l m}^{I}\right|,
$$

prevent the $n l$-potentials from acting on orbitals with a different $l^{\prime}$.

The ingredients in Eq. (3) are produced as follows: A numerical all-electron spin-free relativistic Cowan-GriffinHartree-Fock calculation ${ }^{5}$ is performed on a given state of atom $I$ (usually the ground state, see Tables IV and V). From this calculation, a set of atomic orbitals is arbitrarily defined as the core $\left\{\phi_{c}^{I}\right\}$ (see Table I). The core orbitals are represented by analytical Gaussian expansions which are obtained by a maximum overlap criterion ${ }^{28}$ and they and their orbital energies $\varepsilon_{c}^{I}$ are conveniently stored in libraries. With the numerical core orbitals, the core Coulomb potential $2 \Sigma_{c} \hat{J}_{c}^{I}(r)$ is calculated, $-Z_{\text {core }}^{I} / r$ is added to it, and the result is represented with a local potential $1 / r \Sigma_{k} C_{k}^{I} \exp \left(-\alpha_{k}^{I} r^{2}\right)$ by means of a least-squares fitting procedure and stored. This is the third term on the right-hand side of Eq. (3). The massvelocity and Darwin radial operators of Cowan-GriffinWood-Boring corresponding to the valence orbitals are also stored: $\left\{V_{M V, n l}^{I}+V_{D, n l}^{I}\right\}_{n l \in \text { valence. In a given atomic or mo- }}$ lecular calculation, these scalar relativistic operators are added to the core exchange operator $-\Sigma_{c} \hat{K}_{c}^{I}$ and the result is spectrally represented in the space defined by the set of (Gaussian) primitive functions used for atom $I\left\{\chi_{a l m}^{I}\right\}$, which results in the fourth term on the right-hand side of Eq. (3). In consequence, the $A_{l ; a b}^{I, M P}$ coefficients are the elements of the product matrix $S^{I^{-1}} V_{E M D}^{I} S^{I^{-1}}$; here, $S^{I}$ is the overlap matrix in the basis of primitives $\left\{\chi_{a l m}^{I}\right\}$ and $V_{E M D}^{I}$ is the matrix of the operator $-\Sigma_{c \in \operatorname{core}} \hat{K}_{c}^{I}+\hat{V}_{M V}^{I}+\hat{V}_{D}^{I}$ of atom $I$ in the same basis. Since the set of primitives $\left\{\chi_{a l m}^{I}\right\}$ is likely to change from one molecular calculation to another, and it would not be efficient to tabulate the $A_{l ; a b}^{I, M P}$ coefficients, they are calculated during the input processing part of every single molecular calculation. ${ }^{29}$ Strictly speaking, Eq. (5) holds only when one valence orbital exists for a given $l$, whereas a different operator corresponds when more than one valence orbital of a given $l$ is used; the use of coupling operator techniques ${ }^{30}$ would be indicated in the latter case, leading to an operator which causes, say, the $V_{M V, 5 s}$ to act on the $\phi_{5 s}$ and the $V_{M V, 6 s}$ to act on the $\phi_{6 s}$. It has been shown, however, that using the mass-velocity and Darwin potentials of the outermost valence orbitals for the inner valence orbitals leads to very small errors, safely acceptable within an effective potential method. ${ }^{14}$ As a consequence with this, we will use this approximation. Finally, the last term on the right- 
TABLE II. Valence spin-orbit model potentials [Eq. (9)] of the lanthanide elements.

\begin{tabular}{|c|c|c|c|c|c|}
\hline \multicolumn{2}{|c|}{$V_{\mathrm{SO}, 5 p}$} & \multicolumn{2}{|c|}{$V_{\mathrm{SO}, 5 d}$} & \multicolumn{2}{|c|}{$V_{\mathrm{SO}, 4 f}$} \\
\hline$\beta_{k}^{5 p}$ & $B_{k}^{5 p}$ & $\beta_{k}^{5 d}$ & $B_{k}^{5 d}$ & $\beta_{k}^{4 f}$ & $B_{k}^{4 f}$ \\
\hline \multicolumn{6}{|c|}{ Cerium } \\
\hline 1466000.0 & 0.28398889 & 661400.0 & 0.35302714 & 512000.0 & 0.37087244 \\
\hline 170300.0 & 0.26022545 & 64360.0 & 0.24852100 & 46890.0 & 0.23862575 \\
\hline 29790.0 & 0.17251009 & 8505.0 & 0.12528538 & 5777.7 & 0.11198585 \\
\hline 3960.3 & 0.09561129 & 1232.7 & 0.05415273 & 774.45 & 0.04551961 \\
\hline 476.14 & 0.03972520 & 184.72 & 0.02238901 & 110.649 & 0.01769407 \\
\hline 53.796 & 0.01453447 & 27.374 & 0.00910882 & 17.6163 & 0.00655551 \\
\hline 5.7550 & 0.00397200 & 3.4660 & 0.00263319 & 2.5920 & 0.00199974 \\
\hline 0.5772 & 0.00065578 & 0.4051 & 0.00040643 & 0.2947 & 0.00031300 \\
\hline \multicolumn{6}{|c|}{ Praseodymium } \\
\hline 2195000.0 & 0.27054143 & 668700.0 & 0.34963049 & 508000.0 & 0.36919453 \\
\hline 201300.0 & 0.29586713 & 65730.0 & 0.25002810 & 46790.0 & 0.23986620 \\
\hline 26790.0 & 0.17989171 & 8795.0 & 0.12769819 & 5801.7 & 0.11338803 \\
\hline 3985.8 & 0.09657645 & 1291.6 & 0.05580506 & 783.35 & 0.04628019 \\
\hline 476.14 & 0.04057212 & 195.83 & 0.02325833 & 113.379 & 0.01804460 \\
\hline 53.796 & 0.01473146 & 29.324 & 0.00951809 & 18.3963 & 0.00674120 \\
\hline 5.7550 & 0.00405080 & 3.7640 & 0.00282917 & 2.7480 & 0.00214248 \\
\hline 0.6023 & 0.00065982 & 0.4689 & 0.00045235 & 0.3224 & 0.00032361 \\
\hline \multicolumn{6}{|c|}{ Neodymium } \\
\hline 477000.0 & 0.13586286 & 632200.0 & 0.35125370 & 554000.0 & 0.36101873 \\
\hline 267400.0 & 0.27518520 & 31820.0 & 0.24943424 & 52590.0 & 0.24438379 \\
\hline 26790.0 & 0.19683519 & 8217.0 & 0.12655690 & 6762.7 & 0.11987797 \\
\hline 3960.3 & 0.09564156 & 1196.7 & 0.05503902 & 949.75 & 0.05017133 \\
\hline 476.14 & 0.04139923 & 180.73 & 0.02270305 & 141.179 & 0.02045149 \\
\hline 53.796 & 0.01492464 & 27.424 & 0.00930349 & 21.7463 & 0.00784070 \\
\hline 5.7550 & 0.00413960 & 3.4460 & 0.00277712 & 3.0330 & 0.00242688 \\
\hline 0.5772 & 0.00065362 & 0.3849 & 0.00087968 & 0.3174 & 0.00034314 \\
\hline \multicolumn{6}{|c|}{ Promethium } \\
\hline 2817000.0 & 0.22310125 & 616000.0 & 0.35065727 & 560000.0 & 0.35792076 \\
\hline 251100.0 & 0.33453947 & 60350.0 & 0.24967421 & 53690.0 & 0.24604192 \\
\hline 26190.0 & 0.19237118 & 8048.0 & 0.12690878 & 6986.7 & 0.12223876 \\
\hline 3972.9 & 0.09693086 & 1177.3 & 0.05534218 & 993.65 & 0.05164581 \\
\hline 476.14 & 0.04214806 & 179.25 & 0.02280858 & 149.459 & 0.02124820 \\
\hline 53.796 & 0.01512147 & 27.764 & 0.00939394 & 23.1463 & 0.00822437 \\
\hline 5.7550 & 0.00423137 & 3.5520 & 0.00287812 & 3.2390 & 0.00259707 \\
\hline 0.5792 & 0.00064780 & 0.4016 & 0.00089271 & 0.3404 & 0.00036692 \\
\hline \multicolumn{6}{|c|}{ Samarium } \\
\hline 1625000.0 & 0.26694320 & 612200.0 & 0.34863403 & 565000.0 & 0.35489171 \\
\hline 176200.0 & 0.27960814 & 60330.0 & 0.25065895 & 54690.0 & 0.24752360 \\
\hline 25790.0 & 0.17515724 & 8104.0 & 0.12833309 & 7199.7 & 0.12447164 \\
\hline 4032.6 & 0.10090541 & 1194.7 & 0.05631391 & 1037.05 & 0.05307775 \\
\hline 475.05 & 0.04276649 & 183.43 & 0.02330668 & 157.839 & 0.02204625 \\
\hline 53.737 & 0.01535502 & 28.724 & 0.00964777 & 24.5663 & 0.00861154 \\
\hline 5.7550 & 0.00427055 & 3.7180 & 0.00301801 & 3.4460 & 0.00277128 \\
\hline 0.6112 & 0.00066789 & 0.4250 & 0.00041351 & 0.3627 & 0.00038979 \\
\hline \multicolumn{6}{|c|}{ Europium } \\
\hline 1462000.0 & 0.27214271 & 654800.0 & 0.34101203 & 559000.0 & 0.35316561 \\
\hline 163400.0 & 0.27118727 & 65920.0 & 0.25342086 & 54390.0 & 0.24841341 \\
\hline 25320.0 & 0.17215532 & 9093.0 & 0.13362407 & 7192.7 & 0.12597154 \\
\hline 4016.7 & 0.10201417 & 1879.9 & 0.05995708 & 1041.55 & 0.05380059 \\
\hline 473.79 & 0.04336682 & 216.96 & 0.02543432 & 160.529 & 0.02229714 \\
\hline 53.604 & 0.01565842 & 33.984 & 0.01071905 & 25.7663 & 0.00880202 \\
\hline 5.7550 & 0.00432786 & 4.5030 & 0.00342950 & 3.7440 & 0.00295190 \\
\hline 0.6534 & 0.00067839 & 0.5805 & 0.00055385 & 0.4284 & 0.00043768 \\
\hline \multicolumn{6}{|c|}{ Gadolinium } \\
\hline 1422000.0 & 0.26955738 & 604000.0 & 0.34476808 & 564000.0 & 0.35019807 \\
\hline 162500.0 & 0.26904054 & 60180.0 & 0.25214029 & 55390.0 & 0.24959413 \\
\hline 25410.0 & 0.17417771 & 8197.0 & 0.13105394 & 7421.7 & 0.12785603 \\
\hline 4011.6 & 0.10333387 & 1226.1 & 0.05818757 & 1088.75 & 0.05531791 \\
\hline
\end{tabular}


TABLE II. (Continued.)

\begin{tabular}{|c|c|c|c|c|c|}
\hline \multicolumn{2}{|c|}{$V_{\mathrm{SO}, 5 p}$} & \multicolumn{2}{|c|}{$V_{\mathrm{SO}, 5 d}$} & \multicolumn{2}{|c|}{$V_{\mathrm{SO}, 4 f}$} \\
\hline$\beta_{k}^{5 p}$ & $B_{k}^{5 p}$ & $\beta_{k}^{5 d}$ & $B_{k}^{5 d}$ & $\beta_{k}^{4 f}$ & $B_{k}^{4 f}$ \\
\hline 474.99 & 0.04397859 & 191.44 & 0.02426852 & 468.789 & 0.02324348 \\
\hline 53.963 & 0.01582377 & 30.654 & 0.01014923 & 26.6863 & 0.00920503 \\
\hline 5.7550 & 0.00444481 & 4.0610 & 0.00330970 & 3.7780 & 0.00307651 \\
\hline \multirow[t]{2}{*}{0.6184} & 0.00065864 & 0.4723 & 0.00045411 & 0.3969 & 0.00042153 \\
\hline & \multicolumn{3}{|c|}{ Terbium } & & \\
\hline 409000.0 & 0.26875247 & 600400.0 & 0.34275087 & 559000.0 & 0.34848593 \\
\hline 162500.0 & 0.26518573 & 60150.0 & 0.25289345 & 55190.0 & 0.25025073 \\
\hline 25990.0 & 0.17741281 & 8249.0 & 0.13242312 & 7448.7 & 0.12906227 \\
\hline 4024.3 & 0.10559953 & 1242.9 & 0.05912579 & 1100.95 & 0.05616182 \\
\hline 473.76 & 0.04463014 & 195.84 & 0.02475961 & 172.189 & 0.02398825 \\
\hline 53.820 & 0.01600063 & 31.714 & 0.01041557 & 27.5163 & 0.00943830 \\
\hline 5.7550 & 0.00453073 & 4.2500 & 0.00346460 & 3.9260 & 0.00321686 \\
\hline \multirow[t]{2}{*}{0.6163} & 0.00065010 & 0.4990 & 0.00047812 & 0.4124 & 0.00043565 \\
\hline & & \multicolumn{2}{|l|}{ Dysprosium } & & \\
\hline 1440000.0 & 0.26488999 & 596000.0 & 0.34090483 & 553000.0 & 0.34683795 \\
\hline 166800.0 & 0.26479530 & 60010.0 & 0.25354568 & 54890.0 & 0.25083121 \\
\hline 26280.0 & 0.18396707 & 8286.0 & 0.13366507 & 7455.7 & 0.13017828 \\
\hline 3915.7 & 0.10639408 & 1257.5 & 0.06003476 & 1109.45 & 0.05693361 \\
\hline 464.34 & 0.04460545 & 199.72 & 0.02523220 & 174.969 & 0.02409600 \\
\hline 53.359 & 0.01607689 & 32.694 & 0.01066477 & 28.2563 & 0.00965126 \\
\hline 5.7550 & 0.00460323 & 4.4320 & 0.00361786 & 4.0680 & 0.00335158 \\
\hline \multirow[t]{2}{*}{0.6192} & 0.00064501 & 0.5242 & 0.00050023 & 0.4280 & 0.00044899 \\
\hline & & \multicolumn{2}{|c|}{ Holmium } & & \\
\hline 1480000.0 & 0.25969939 & 593900.0 & 0.33877309 & 547000.0 & 0.34541340 \\
\hline 171600.0 & 0.26647573 & 60130.0 & 0.25430843 & 54490.0 & 0.25145341 \\
\hline 26420.0 & 0.18941966 & 8360.0 & 0.13510571 & 7440.7 & 0.13119725 \\
\hline 3839.7 & 0.10712948 & 1277.7 & 0.06106446 & 1113.15 & 0.05765278 \\
\hline 458.45 & 0.04480387 & 204.41 & 0.02576813 & 176.679 & 0.02444615 \\
\hline 53.097 & 0.01618689 & 33.764 & 0.01093877 & 28.8463 & 0.00982594 \\
\hline 5.7830 & 0.00468414 & 4.6230 & 0.00377889 & 4.2000 & 0.00347849 \\
\hline \multirow[t]{2}{*}{0.62710} & 0.00064494 & 0.5500 & 0.00052288 & 0.4431 & 0.00046115 \\
\hline & & \multicolumn{2}{|c|}{ Erbium } & & \\
\hline 1491000.0 & 0.25708555 & 586000.0 & 0.33743322 & 428000.0 & 0.36095602 \\
\hline 172800.0 & 0.26642553 & 59540.0 & 0.25474991 & 40590.0 & 0.24492287 \\
\hline 26480.0 & 0.19260976 & 8318.0 & 0.13598283 & 5163.7 & 0.11990360 \\
\hline 3803.4 & 0.10811181 & 1278.1 & 0.06170639 & 718.05 & 0.05045285 \\
\hline 456.42 & 0.04523341 & 206.07 & 0.02608754 & 104.429 & 0.02001341 \\
\hline 53.356 & 0.01630755 & 34.494 & 0.01112103 & 17.0263 & 0.00704562 \\
\hline 5.9490 & 0.00481688 & 4.7890 & 0.00392281 & 2.9330 & 0.00249422 \\
\hline \multirow[t]{2}{*}{0.6519} & 0.00066698 & 0.5728 & 0.00054170 & 0.3205 & 0.00030240 \\
\hline & & \multicolumn{2}{|l|}{ Thullium } & & \\
\hline 1474000.0 & 0.25579415 & 583800.0 & 0.33537248 & 423000.0 & 0.35995079 \\
\hline 173400.0 & 0.26250475 & 59620.0 & 0.25543972 & 40190.0 & 0.24575141 \\
\hline 26790.0 & 0.19732029 & 8884.0 & 0.13735168 & 5162.7 & 0.12075865 \\
\hline 3748.3 & 0.10912081 & 1296.8 & 0.06271707 & 715.95 & 0.05100441 \\
\hline 456.41 & 0.04534768 & 210.45 & 0.02661041 & 104.249 & 0.02029715 \\
\hline 54.564 & 0.01658041 & 35.534 & 0.01138581 & 16.9163 & 0.00712099 \\
\hline 6.1530 & 0.00499767 & 4.9830 & 0.00408661 & 2.9640 & 0.00252365 \\
\hline \multirow[t]{2}{*}{0.6769} & 0.00068941 & 0.5988 & 0.00056418 & 0.3243 & 0.00030197 \\
\hline & & \multicolumn{2}{|l|}{ Ytterbium } & & \\
\hline 1428000.0 & 0.25457853 & 578400.0 & 0.33375839 & 417000.0 & 0.35879243 \\
\hline 170800.0 & 0.26093012 & 59310.0 & 0.25593169 & 39790.0 & 0.24630181 \\
\hline 26790.0 & 0.19626670 & 8386.0 & 0.13840533 & 5136.7 & 0.12160026 \\
\hline 3823.6 & 0.11087297 & 1304.5 & 0.06350264 & 715.25 & 0.05160595 \\
\hline 462.97 & 0.04663480 & 213.24 & 0.02700822 & 104.199 & 0.02059842 \\
\hline 54.504 & 0.01683657 & 36.414 & 0.01160464 & 16.8163 & 0.00720472 \\
\hline 6.1730 & 0.00507772 & 5.1670 & 0.00424507 & 2.9960 & 0.00255117 \\
\hline 0.6824 & 0.00068579 & 0.6236 & 0.00058467 & 0.3278 & 0.00030158 \\
\hline
\end{tabular}


TABLE II. (Continued.)

\begin{tabular}{|c|c|c|c|c|c|}
\hline \multicolumn{2}{|c|}{$V_{\mathrm{SO}, 5 p}$} & \multicolumn{2}{|c|}{$V_{\mathrm{SO}, 5 d}$} & \multicolumn{2}{|c|}{$V_{\mathrm{SO}, 4 f}$} \\
\hline$\beta_{k}^{5 p}$ & $B_{k}^{5 p}$ & $\beta_{k}^{5 d}$ & $B_{k}^{5 d}$ & $\beta_{k}^{4 f}$ & $B_{k}^{4 f}$ \\
\hline \multicolumn{6}{|c|}{ Lutetium } \\
\hline 1498000.0 & 0.25025448 & 574900.0 & 0.33188060 & 369000.0 & 0.36538115 \\
\hline 174800.0 & 0.26440986 & 59230.0 & 0.25648311 & 34390.0 & 0.24299740 \\
\hline 26790.0 & 0.20054499 & 8425.0 & 0.13961049 & 4817.7 & 0.11666403 \\
\hline 3769.7 & 0.11139124 & 1318.4 & 0.06443015 & 582.05 & 0.04863788 \\
\hline 462.17 & 0.04690647 & 216.86 & 0.02747021 & 80.269 & 0.01917173 \\
\hline 55.095 & 0.01706387 & 37.404 & 0.01185019 & 11.2563 & 0.00689882 \\
\hline 6.2910 & 0.00522307 & 5.3620 & 0.00441303 & 1.9090 & 0.00167062 \\
\hline 0.6927 & 0.00069085 & 0.6496 & 0.00060595 & 0.1905 & 0.00015526 \\
\hline
\end{tabular}

TABLE III. Valence spin-orbit model potentials [Eq. (9)] of the actinide elements.

\begin{tabular}{|c|c|c|c|c|c|}
\hline \multicolumn{2}{|c|}{$V_{\mathrm{SO}, 6 p}$} & \multicolumn{2}{|c|}{$V_{\mathrm{SO}, 6 d}$} & \multicolumn{2}{|c|}{$V_{\mathrm{SO}, 5 f}$} \\
\hline$\beta_{k}^{6 p}$ & $B_{k}^{6 p}$ & $\beta_{k}^{6 d}$ & $B_{k}^{6 d}$ & $\beta_{k}^{5 f}$ & $B_{k}^{5 f}$ \\
\hline \multicolumn{6}{|c|}{ Thorium } \\
\hline 2115500.0 & 0.18807358 & 397210.0 & 0.32240593 & 339700.0 & 0.33531520 \\
\hline 239900.0 & 0.25863146 & 41795.0 & 0.25988923 & 34730.0 & 0.25631360 \\
\hline 31155.0 & 0.26576843 & 6130.4 & 0.14556945 & 4901.1 & 0.13776822 \\
\hline 3567.40 & 0.13419529 & 965.42 & 0.07107308 & 751.77 & 0.06365193 \\
\hline 478.251 & 0.05645098 & 148.737 & 0.03026420 & 116.351 & 0.02730631 \\
\hline 61.5265 & 0.02170885 & 23.1840 & 0.01214338 & 17.0973 & 0.01063654 \\
\hline 8.16690 & 0.00713323 & 3.64360 & 0.00337634 & 2.51790 & 0.00247125 \\
\hline 0.75664 & 0.00109121 & 0.45163 & 0.00054379 & 0.29921 & 0.00035064 \\
\hline \multicolumn{6}{|c|}{ Protactinium } \\
\hline 2262800.0 & 0.18386282 & 403800.0 & 0.31938595 & 341300.0 & 0.33313647 \\
\hline 251660.0 & 0.25709424 & 42804.0 & 0.26022825 & 35090.0 & 0.25694965 \\
\hline 32152.0 & 0.27196012 & 6323.5 & 0.14834146 & 4989.9 & 0.13918498 \\
\hline 3586.19 & 0.13670020 & 999.91 & 0.07209771 & 771.98 & 0.06474262 \\
\hline 477.723 & 0.05704302 & 158.464 & 0.03111940 & 120.479 & 0.02793512 \\
\hline 61.6452 & 0.02182383 & 25.1450 & 0.01265997 & 17.8783 & 0.01096387 \\
\hline 8.40710 & 0.00720902 & 4.09620 & 0.00366669 & 2.66110 & 0.00259277 \\
\hline 0.81311 & 0.00117508 & 0.53249 & 0.00065391 & 0.34180 & 0.00039338 \\
\hline \multicolumn{6}{|c|}{ Uranium } \\
\hline 3455000.0 & 0.16411390 & 406000.0 & 0.31688834 & 340000.0 & 0.33182698 \\
\hline 341300.0 & 0.25113771 & 43800.0 & 0.26068266 & 34990.0 & 0.25783825 \\
\hline 39680.0 & 0.29933541 & 6445.0 & 0.14959338 & 4971.7 & 0.14020204 \\
\hline 3915.50 & 0.14917883 & 1040.60 & 0.07269406 & 768.85 & 0.06527392 \\
\hline 473.940 & 0.06057388 & 171.020 & 0.03210768 & 119.919 & 0.02819271 \\
\hline 55.0220 & 0.02148873 & 27.3740 & 0.01344723 & 17.6863 & 0.01109856 \\
\hline 7.55000 & 0.00659643 & 4.34800 & 0.00397025 & 2.51500 & 0.00257394 \\
\hline 0.74710 & 0.00108180 & 0.53540 & 0.00068187 & 0.30080 & 0.00035306 \\
\hline \multicolumn{6}{|c|}{ Neptunium } \\
\hline 2684200.0 & 0.16677019 & 395030.0 & 0.31714267 & 334900.0 & 0.33104187 \\
\hline 301410.0 & 0.25070113 & 42106.0 & 0.26087349 & 34620.0 & 0.25762556 \\
\hline 36260.0 & 0.29078023 & 6259.0 & 0.14912354 & 4955.5 & 0.14051541 \\
\hline 3743.00 & 0.14490014 & 1007.84 & 0.07288759 & 773.35 & 0.06576544 \\
\hline 485.113 & 0.05884013 & 163.647 & 0.03179686 & 121.664 & 0.02854876 \\
\hline 62.3540 & 0.02245398 & 26.3570 & 0.01380857 & 18.1073 & 0.01131842 \\
\hline 8.54110 & 0.00785335 & 4.16580 & 0.00384281 & 2.57700 & 0.00255209 \\
\hline 0.83689 & 0.00123686 & 0.53611 & 0.00067354 & 0.31743 & 0.00087180 \\
\hline \multicolumn{6}{|c|}{ Plutonium } \\
\hline 3146300.0 & 0.15705658 & 397190.0 & 0.31481803 & 335300.0 & 0.32916581 \\
\hline 342500.0 & 0.24717359 & 42552.0 & 0.26128841 & 34820.0 & 0.25816493 \\
\hline 39226.0 & 0.30382413 & 6366.4 & 0.15054651 & 5013.1 & 0.14172039 \\
\hline 3816.52 & 0.15005055 & 1032.92 & 0.07400642 & 787.62 & 0.06668599 \\
\hline 480.956 & 0.05980538 & 169.170 & 0.03249400 & 124.723 & 0.02908489 \\
\hline 60.8175 & 0.02247679 & 27.3740 & 0.01371061 & 18.6923 & 0.01160200 \\
\hline 8.43470 & 0.00725165 & 4.32630 & 0.00398124 & 2.66200 & 0.00275555 \\
\hline 0.85137 & 0.00125980 & 0.56901 & 0.00071962 & 0.33557 & 0.00039269 \\
\hline
\end{tabular}


TABLE III. (Continued.)

\begin{tabular}{|c|c|c|c|c|c|}
\hline \multicolumn{2}{|l|}{$V_{\mathrm{SO}, 6 p}$} & \multicolumn{2}{|c|}{$V_{\mathrm{SO}, 6 d}$} & \multicolumn{2}{|c|}{$V_{\mathrm{SO}, 5 f}$} \\
\hline$\beta_{k}^{6 p}$ & $B_{k}^{6 p}$ & $\beta_{k}^{6 d}$ & $B_{k}^{6 d}$ & $\beta_{k}^{5 f}$ & $B_{k}^{5 f}$ \\
\hline \multicolumn{6}{|c|}{ Americium } \\
\hline 3384200.0 & 0.15574777 & 405340.0 & 0.31127696 & 342800.0 & 0.32553576 \\
\hline 350590.0 & 0.24668424 & 43751.0 & 0.26187097 & 35910.0 & 0.25905123 \\
\hline 39680.0 & 0.30725319 & 6573.9 & 0.15357974 & 5226.9 & 0.14403557 \\
\hline 3816.22 & 0.15158511 & 1055.52 & 0.07596999 & 830.51 & 0.06847034 \\
\hline 478.783 & 0.06061052 & 170.635 & 0.03315827 & 132.957 & 0.03010085 \\
\hline 59.8045 & 0.02251656 & 27.3740 & 0.01390707 & 20.2453 & 0.01210738 \\
\hline 8.51280 & 0.00718815 & 4.33300 & 0.00394757 & 3.00620 & 0.00299447 \\
\hline 0.90958 & 0.00133541 & 0.62397 & 0.00076651 & 0.42026 & 0.00048780 \\
\hline \multicolumn{6}{|c|}{ Curium } \\
\hline 3819100.0 & 0.15493299 & 398590.0 & 0.31094423 & 335300.0 & 0.32557711 \\
\hline 875530.0 & 0.23924896 & 43111.0 & 0.26138934 & 35130.0 & 0.25909496 \\
\hline 42280.0 & 0.31583539 & 6573.7 & 0.15268259 & 5113.4 & 0.14397822 \\
\hline 3915.50 & 0.15622384 & 1072.33 & 0.07732694 & 813.74 & 0.06844211 \\
\hline 475.913 & 0.06242639 & 170.720 & 0.03373706 & 130.472 & 0.03011601 \\
\hline 56.2405 & 0.02257115 & 27.3740 & 0.01401285 & 19.8133 & 0.01215731 \\
\hline 7.87250 & 0.00682268 & 4.29590 & 0.00400079 & 2.81030 & 0.00296310 \\
\hline 0.85401 & 0.00125265 & 0.60138 & 0.00074924 & 0.36567 & 0.00042461 \\
\hline \multicolumn{6}{|c|}{ Berkelium } \\
\hline 6089100.0 & 0.25814589 & 347780.0 & 0.32075276 & 335000.0 & 0.32388997 \\
\hline 242300.0 & 0.21846954 & 37509.0 & 0.25466083 & 35240.0 & 0.25950851 \\
\hline 40780.0 & 0.29503979 & 6025.4 & 0.14304610 & 5155.9 & 0.14502704 \\
\hline 3910.45 & 0.15736507 & 1052.90 & 0.07560481 & 825.59 & 0.06927444 \\
\hline 473.840 & 0.06321337 & 170.678 & 0.03401236 & 133.175 & 0.03061189 \\
\hline 54.9980 & 0.02279069 & 27.3740 & 0.01417216 & 20.3543 & 0.01242880 \\
\hline 7.51950 & 0.00673357 & 4.24660 & 0.00402560 & 2.87810 & 0.00306733 \\
\hline 0.80481 & 0.01118139 & 0.60158 & 0.00074248 & 0.37817 & 0.00043748 \\
\hline \multicolumn{6}{|c|}{ Californium } \\
\hline 3637100.0 & 0.15342975 & 357320.0 & 0.31726836 & 335100.0 & 0.32210082 \\
\hline 667330.0 & 0.23656105 & 39211.0 & 0.25239897 & 35400.0 & 0.25992477 \\
\hline 41380.0 & 0.31767236 & 6416.6 & 0.14893454 & 5206.7 & 0.14613111 \\
\hline 3829.45 & 0.15659807 & 1071.55 & 0.07857249 & 838.98 & 0.07015209 \\
\hline 476.002 & 0.06287879 & 171.020 & 0.03435781 & 136.170 & 0.03113406 \\
\hline 57.2560 & 0.02311452 & 27.3740 & 0.01442292 & 20.9533 & 0.01271424 \\
\hline 8.12690 & 0.00699285 & 4.09850 & 0.0040 .3452 & 2.95920 & 0.00318169 \\
\hline 0.91920 & 0.00135604 & 0.58236 & 0.00070242 & 0.39329 & 0.00045321 \\
\hline \multicolumn{6}{|c|}{ Einstenium } \\
\hline 864700.0 & 0.23983841 & 479390.0 & 0.30620843 & 335800.0 & 0.32020883 \\
\hline 105570.0 & 0.25178512 & 44082.0 & 0.28988964 & 35620.0 & 0.26037921 \\
\hline 17535.0 & 0.20860051 & 5865.0 & 0.15100253 & 5266.7 & 0.14730461 \\
\hline 2893.02 & 0.10874543 & 1045.60 & 0.07449637 & 853.99 & 0.07108153 \\
\hline 538.374 & 0.05797920 & 171.020 & 0.03507326 & 139.457 & 0.03168585 \\
\hline 77.4816 & 0.02614203 & 27.3740 & 0.01426361 & 21.6073 & 0.01301394 \\
\hline 10.71110 & 0.00888466 & 4.31800 & 0.00416817 & 3.05160 & 0.00330561 \\
\hline 1.11645 & 0.00172830 & 0.60904 & 0.00075129 & 0.41037 & 0.00047119 \\
\hline \multicolumn{6}{|c|}{ Fermium } \\
\hline 877200.0 & 0.23674249 & 546370.0 & 0.29523244 & 336000.0 & 0.31837539 \\
\hline 107680.0 & 0.25057496 & 48038.0 & 0.30429666 & 35800.0 & 0.26073001 \\
\hline 17984.0 & 0.21041691 & 5875.0 & 0.15661750 & 5321.8 & 0.14840793 \\
\hline 2978.00 & 0.11058260 & 1040.60 & 0.07425566 & 868.42 & 0.07197888 \\
\hline 554.814 & 0.05983957 & 171.020 & 0.03553503 & 142.703 & 0.03222696 \\
\hline 79.6579 & 0.02679070 & 27.3740 & 0.01430383 & 22.2673 & 0.01331260 \\
\hline 11.01540 & 0.00909760 & 4.34800 & 0.00424917 & 3.14530 & 0.00343227 \\
\hline 1.16229 & 0.00180633 & 0.60816 & 0.00074872 & 0.42761 & 0.00048901 \\
\hline \multicolumn{6}{|c|}{ Mendelevium } \\
\hline 889000.0 & 0.23376131 & 418840.0 & 0.30968001 & 336400.0 & 0.31654428 \\
\hline 109710.0 & 0.24931839 & 40736.0 & 0.27820782 & 35990.0 & 0.26109364 \\
\hline 18427.0 & 0.21209808 & 5857.0 & 0.14894510 & 5377.7 & 0.14951423 \\
\hline 3064.23 & 0.11237097 & 1052.50 & 0.07657507 & 882.98 & 0.07288034 \\
\hline
\end{tabular}


TABLE III. (Continued.)

\begin{tabular}{|c|c|c|c|c|c|}
\hline \multicolumn{2}{|c|}{$V_{\mathrm{SO}, 6 p}$} & \multicolumn{2}{|c|}{$V_{\mathrm{SO}, 6 d}$} & \multicolumn{2}{|c|}{$V_{\mathrm{SO}, 5 f}$} \\
\hline$\beta_{k}^{6 p}$ & $B_{k}^{6 p}$ & $\beta_{k}^{6 d}$ & $B_{k}^{6 d}$ & $\beta_{k}^{5 f}$ & $B_{k}^{5 f}$ \\
\hline 571.698 & 0.06072083 & 171.010 & 0.03579381 & 145.976 & 0.03277190 \\
\hline 81.8691 & 0.02745259 & 27.3740 & 0.01453602 & 22.9343 & 0.01361356 \\
\hline 11.31950 & 0.00931170 & 4.21030 & 0.00426301 & 3.23850 & 0.00356177 \\
\hline 1.20876 & 0.00188510 & 0.59249 & 0.00070978 & 0.44425 & 0.00050577 \\
\hline \multicolumn{6}{|c|}{ Nobelium } \\
\hline 904900.0 & 0.23042955 & 506080.0 & 0.30125016 & 336600.0 & 0.31478595 \\
\hline 112310.0 & 0.24793864 & 45097.0 & 0.29528659 & 36150.0 & 0.26142718 \\
\hline 18950.0 & 0.21416100 & 5915.0 & 0.15556993 & 5428.4 & 0.15056851 \\
\hline 3156.60 & 0.11437522 & 1047.63 & 0.07653736 & 896.69 & 0.07375087 \\
\hline 588.749 & 0.06214647 & 171.020 & 0.03624188 & 149.123 & 0.03330243 \\
\hline 84.0324 & 0.02811455 & 27.3740 & 0.01456305 & 23.5863 & 0.01391001 \\
\hline 11.61560 & 0.00952057 & 4.26790 & 0.00433981 & 3.32610 & 0.00369187 \\
\hline 1.25570 & 0.00196386 & 0.60346 & 0.00072074 & 0.45893 & 0.00051959 \\
\hline \multicolumn{6}{|c|}{ Laurentium } \\
\hline 918700.0 & 0.22733528 & 411420.0 & 0.30933125 & 336200.0 & 0.31319705 \\
\hline 114660.0 & 0.24652857 & 40327.0 & 0.27499181 & 36230.0 & 0.26172319 \\
\hline 19145.0 & 0.21594207 & 5945.0 & 0.15234729 & 5464.6 & 0.15150931 \\
\hline 3249.24 & 0.11625813 & 1040.60 & 0.07812304 & 907.81 & 0.07453516 \\
\hline 606.358 & 0.06359030 & 171.020 & 0.03610723 & 151.834 & 0.03378741 \\
\hline 86.2513 & 0.02879356 & 27.3740 & 0.01500214 & 24.1713 & 0.01418771 \\
\hline 11.91340 & 0.00973198 & 4.00100 & 0.00427289 & 3.39630 & 0.00381658 \\
\hline 1.30365 & 0.00204370 & 0.58883 & 0.00066260 & 0.46866 & 0.00052643 \\
\hline
\end{tabular}

hand side of Eq. (3), which results from the linearindependency conditions between core and valence orbitals ${ }^{2}$ is calculated with the core orbitals and orbital energies. All this defines the spin-free CG-AIMP Hamiltonian [Eq. (2)] which is obtained without resorting to any parametrization procedure based on the use of the valence orbitals.

The spin-orbit contribution in Eq. (1) is

$$
\hat{H}^{\mathrm{SO}}=\sum_{i}^{N_{\text {val }}} \sum_{I}^{N_{\text {nuc }}} \hat{h}_{\mathrm{SO}}^{I}(i),
$$

with the atomic one-electron spin-orbit terms

$\mathrm{Sm}$

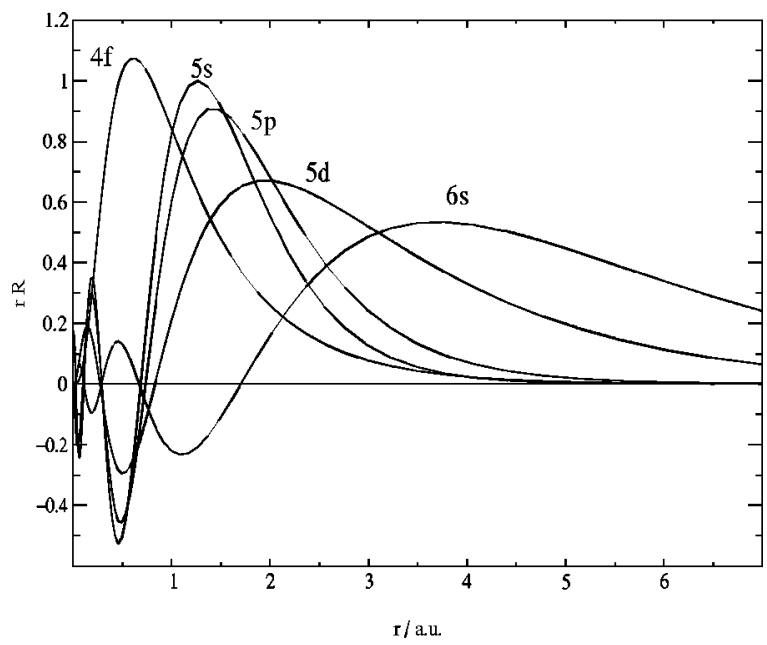

FIG. 1. Valence orbitals of Sm. Full line: numerical Cowan-Griffin allelectron results. Dashed line: analytical AIMP results of this work.

$$
\hat{h}_{\mathrm{SO}}^{I}(i)=\lambda^{I} \sum_{n l \in \text { valence }} V_{\mathrm{SO}, n l}^{I, M P}\left(r_{i}\right) \hat{O}_{l}^{I} \hat{\mathbf{l}} \hat{\mathbf{s}}^{\hat{\mathbf{S}}} \hat{O}_{l}^{I}
$$

Herein, $\hat{\mathbf{l}}^{I}$ and $\hat{\mathbf{s}}$ are the usual vector angular momentum and spin operators, respectively, and the angular projectors $\hat{O}_{l}^{I}$ are used in the form proposed by Pitzer and Winter. ${ }^{31}$ The radial components are chosen to be analytical functions

$$
V_{\mathrm{SO}, n l}^{I, M P}\left(r_{i}\right)=\sum_{k} \frac{B_{k}^{n l, I} \exp \left(-\beta_{k}^{n l, I} r_{i}^{2}\right)}{r_{i}^{2}},
$$

$\mathrm{Pu}$

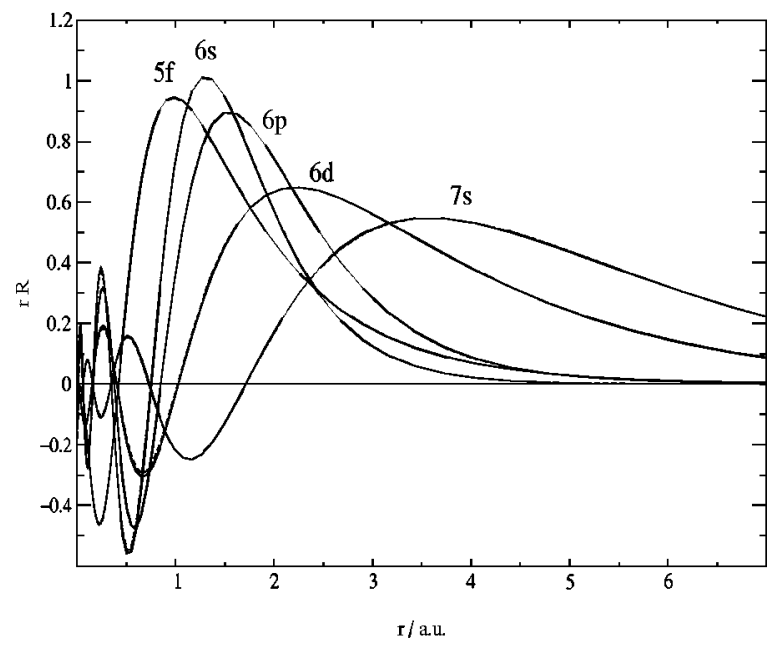

FIG. 2. Valence orbitals of Pu. Full line: numerical Cowan-Griffin allelectron results. Dashed line: analytical AIMP results of this work. 
TABLE IV. Spin-free relativistic valence energy, orbital energies, radial expectation values (in atomic units), and orbital spin-orbit coupling constants $\zeta_{n l}$ (in $\mathrm{cm}^{-1}$ ). The $\zeta_{n l}$ are calculated with the Gaussian orbitals and the analytical operators (Table II) in the CG-AIMP calculations, and with the numerical orbitals and operators [Eq. (10)] in the all-electron numerical Cowan-Griffin-Hartree-Fock calculations. The AIMP calculations correspond to a [Kr,4d] core and a $5 s, 5 p, 4 f, 5 d, 6 s$-valence.

\begin{tabular}{|c|c|c|c|c|c|c|c|c|c|}
\hline & \multicolumn{3}{|c|}{ CG-AIMP } & \multicolumn{3}{|c|}{$\begin{array}{l}\text { Spin-orbit corrected } \\
\text { CG-AIMP }\end{array}$} & \multicolumn{3}{|c|}{$\begin{array}{c}\text { All-electron } \\
\text { numerical CG-HF }\end{array}$} \\
\hline & $\begin{array}{l}\mathrm{E}(\mathrm{val}) \\
\epsilon(5 s) \\
\epsilon(5 p) \\
\epsilon(4 f) \\
\epsilon(5 d) \\
\epsilon(6 s)\end{array}$ & $\begin{array}{l}\langle r\rangle_{5 s} \\
\langle r\rangle_{5 p} \\
\langle r\rangle_{4 f} \\
\langle r\rangle_{5 d} \\
\langle r\rangle_{6 s}\end{array}$ & $\begin{array}{l}\zeta_{5 p} \\
\zeta_{4 f} \\
\zeta_{5 d}\end{array}$ & $\begin{array}{l}\mathrm{E}(\mathrm{val}) \\
\epsilon(5 s) \\
\epsilon(5 p) \\
\epsilon(4 f) \\
\epsilon(5 d) \\
\epsilon(6 s)\end{array}$ & $\begin{array}{l}\langle r\rangle_{5 s} \\
\langle r\rangle_{5 p} \\
\langle r\rangle_{4 f} \\
\langle r\rangle_{5 d} \\
\langle r\rangle_{6 s}\end{array}$ & $\begin{array}{l}\zeta_{5 p} \\
\zeta_{4 f} \\
\zeta_{5 d}\end{array}$ & $\begin{array}{l}\epsilon(5 s) \\
\epsilon(5 p) \\
\epsilon(4 f) \\
\epsilon(5 d) \\
\epsilon(6 s)\end{array}$ & $\begin{array}{l}\langle r\rangle_{5 s} \\
\langle r\rangle_{5 p} \\
\langle r\rangle_{4 f} \\
\langle r\rangle_{5 d} \\
\langle r\rangle_{6 s}\end{array}$ & $\begin{array}{l}\zeta_{5 p} \\
\zeta_{4 f} \\
\zeta_{5 d}\end{array}$ \\
\hline $\mathrm{Ce} \quad\left(f^{1} d^{2} s^{1}\right)^{5} I$ & $\begin{array}{l}-38.368911 \\
-1.8071 \\
-1.0133 \\
-0.4581 \\
-0.2056 \\
-0.1800\end{array}$ & $\begin{array}{l}1.541 \\
1.783 \\
1.090 \\
3.026 \\
4.759\end{array}$ & $\begin{array}{r}12790 \\
747 \\
525\end{array}$ & $\begin{array}{c}-38.367880 \\
-1.8071 \\
-1.0131 \\
-0.4581 \\
-0.2054 \\
-0.1800\end{array}$ & $\begin{array}{l}1.541 \\
1.783 \\
1.090 \\
3.026 \\
4.759\end{array}$ & $\begin{array}{r}12482 \\
748 \\
548\end{array}$ & $\begin{array}{l}-1.8036 \\
-1.0144 \\
-0.4571 \\
-0.2059 \\
-0.1796\end{array}$ & $\begin{array}{l}1.543 \\
1.782 \\
1.091 \\
3.023 \\
4.764\end{array}$ & $\begin{array}{r}12482 \\
748 \\
548\end{array}$ \\
\hline $\operatorname{Pr}\left(f^{3} d^{1} s^{1}\right)^{6} L$ & $\begin{array}{l}-46.931387 \\
-1.7258 \\
-0.9287 \\
-0.3275 \\
-0.1591 \\
-0.1654\end{array}$ & $\begin{array}{l}1.541 \\
1.800 \\
1.099 \\
3.378 \\
5.016\end{array}$ & $\begin{array}{r}12829 \\
807 \\
427\end{array}$ & $\begin{array}{c}-46.929913 \\
-1.7258 \\
-0.9285 \\
-0.3274 \\
-0.1587 \\
-0.1654\end{array}$ & $\begin{array}{l}1.541 \\
1.800 \\
1.099 \\
3.378 \\
5.016\end{array}$ & $\begin{array}{r}12465 \\
809 \\
465\end{array}$ & $\begin{array}{l}-1.7228 \\
-0.9302 \\
-0.3271 \\
-0.1597 \\
-0.1651\end{array}$ & $\begin{array}{l}1.541 \\
1.798 \\
1.100 \\
3.374 \\
5.025\end{array}$ & $\begin{array}{r}12465 \\
809 \\
464\end{array}$ \\
\hline $\mathrm{Nd} \quad\left(f^{3} d^{1} s^{2}\right)^{5} K$ & $\begin{array}{l}-56.625703 \\
-2.0040 \\
-1.1363 \\
-0.6949 \\
-0.2592 \\
-0.1864\end{array}$ & $\begin{array}{l}1.468 \\
1.698 \\
0.947 \\
2.658 \\
4.538\end{array}$ & $\begin{array}{r}15354 \\
1068 \\
775\end{array}$ & $\begin{array}{c}-56.623937 \\
-2.0040 \\
-1.1360 \\
-0.6949 \\
-0.2592 \\
-0.1864\end{array}$ & $\begin{array}{l}1.468 \\
1.698 \\
0.947 \\
2.658 \\
4.538\end{array}$ & $\begin{array}{r}14845 \\
1072 \\
795\end{array}$ & $\begin{array}{l}-2.0005 \\
-1.1379 \\
-0.6951 \\
-0.2600 \\
-0.1861\end{array}$ & $\begin{array}{l}1.469 \\
1.697 \\
0.946 \\
2.655 \\
4.544\end{array}$ & $\begin{array}{r}14846 \\
1072 \\
795\end{array}$ \\
\hline $\operatorname{Pm} \quad\left(f^{4} d^{1} s^{2}\right)^{6} L$ & $\begin{array}{c}-67.873986 \\
-2.0603 \\
-1.1614 \\
-0.7344 \\
-0.2638 \\
-0.1885\end{array}$ & $\begin{array}{l}1.438 \\
1.666 \\
0.911 \\
2.603 \\
4.483\end{array}$ & $\begin{array}{r}16586 \\
1226 \\
849\end{array}$ & $\begin{array}{c}-67.871695 \\
-2.0603 \\
-1.1611 \\
-0.7344 \\
-0.2637 \\
-0.1885\end{array}$ & $\begin{array}{l}1.438 \\
1.666 \\
0.910 \\
2.603 \\
4.483\end{array}$ & $\begin{array}{r}15973 \\
1229 \\
870\end{array}$ & $\begin{array}{l}-2.0574 \\
-1.1630 \\
-0.7346 \\
-0.2646 \\
-0.1884\end{array}$ & $\begin{array}{l}1.439 \\
1.665 \\
0.910 \\
2.600 \\
4.489\end{array}$ & $\begin{array}{r}15974 \\
1230 \\
870\end{array}$ \\
\hline $\operatorname{Sm}\left(f^{5} d^{1} s^{2}\right)^{7} K$ & $\begin{array}{c}-80.618805 \\
-2.1160 \\
-1.1868 \\
-0.7599 \\
-0.2660 \\
-0.1908\end{array}$ & $\begin{array}{l}1.410 \\
1.636 \\
0.879 \\
2.564 \\
4.430\end{array}$ & $\begin{array}{r}17887 \\
1394 \\
918\end{array}$ & $\begin{array}{c}-80.615943 \\
-2.1161 \\
-1.1864 \\
-0.7600 \\
-0.2660 \\
-0.1908\end{array}$ & $\begin{array}{l}1.410 \\
1.636 \\
0.879 \\
2.564 \\
4.430\end{array}$ & $\begin{array}{r}17154 \\
1398 \\
926\end{array}$ & $\begin{array}{l}-2.1150 \\
-1.1881 \\
-0.7597 \\
-0.2665 \\
-0.1908\end{array}$ & $\begin{array}{l}1.410 \\
1.635 \\
0.879 \\
2.562 \\
4.433\end{array}$ & $\begin{array}{r}17154 \\
1398 \\
926\end{array}$ \\
\hline Eu $\quad\left(f^{7} d^{1} s^{1}\right){ }^{10} D$ & $\begin{array}{c}-95.310839 \\
-1.9421 \\
-1.0258 \\
-0.4688 \\
-0.1378 \\
-0.1811\end{array}$ & $\begin{array}{l}1.421 \\
1.666 \\
0.903 \\
3.521 \\
4.682\end{array}$ & $\begin{array}{r}17549 \\
1459 \\
469\end{array}$ & $\begin{array}{l}-95.307573 \\
-1.9422 \\
-1.0253 \\
-0.4689 \\
-0.1377 \\
-0.1811\end{array}$ & $\begin{array}{l}1.421 \\
1.666 \\
0.903 \\
3.521 \\
4.682\end{array}$ & $\begin{array}{r}16761 \\
1462 \\
481\end{array}$ & $\begin{array}{l}-1.9459 \\
-1.0269 \\
-0.4680 \\
-0.1382 \\
-0.1812\end{array}$ & $\begin{array}{l}1.417 \\
1.665 \\
0.904 \\
3.526 \\
4.685\end{array}$ & $\begin{array}{r}16760 \\
1462 \\
481\end{array}$ \\
\hline Gd $\quad\left(f^{7} d^{1} s^{2}\right)^{9} D$ & $\begin{array}{c}-111.099477 \\
-2.2300 \\
-1.2393 \\
-0.8353 \\
-0.2447 \\
-0.1969\end{array}$ & $\begin{array}{l}1.358 \\
1.580 \\
0.821 \\
2.591 \\
4.312\end{array}$ & $\begin{array}{r}20685 \\
1778 \\
952\end{array}$ & $\begin{array}{c}-111.094954 \\
-2.2300 \\
-1.2387 \\
-0.8354 \\
-0.2447 \\
-0.1969\end{array}$ & $\begin{array}{l}1.358 \\
1.580 \\
0.821 \\
2.591 \\
4.312\end{array}$ & $\begin{array}{r}19657 \\
1780 \\
950\end{array}$ & $\begin{array}{l}-2.2336 \\
-1.2400 \\
-0.8339 \\
-0.2447 \\
-0.1971\end{array}$ & $\begin{array}{l}1.357 \\
1.579 \\
0.822 \\
2.591 \\
4.310\end{array}$ & $\begin{array}{r}19656 \\
1780 \\
950\end{array}$ \\
\hline $\mathrm{Tb} \quad\left(f^{8} d^{1} s^{2}\right)^{8} H$ & $\begin{array}{c}-128.631426 \\
-2.2989 \\
-1.2721 \\
-0.8135 \\
-0.2267 \\
-0.2010\end{array}$ & $\begin{array}{l}1.332 \\
1.551 \\
0.803 \\
0.648 \\
4.241\end{array}$ & $\begin{array}{r}22309 \\
1974 \\
919\end{array}$ & $\begin{array}{c}-128.625804 \\
-2.2989 \\
-1.2712 \\
-0.8136 \\
-0.2267 \\
-0.2010\end{array}$ & $\begin{array}{l}1.332 \\
1.551 \\
0.803 \\
2.648 \\
4.241\end{array}$ & $\begin{array}{r}21104 \\
1978 \\
926\end{array}$ & $\begin{array}{l}-2.3033 \\
-1.2726 \\
-0.8120 \\
-0.2266 \\
-0.2014\end{array}$ & $\begin{array}{l}1.330 \\
1.550 \\
0.804 \\
2.649 \\
4.238\end{array}$ & $\begin{array}{r}21102 \\
1978 \\
926\end{array}$ \\
\hline Dy $\left(f^{9} d^{1} s^{2}\right)^{7} K$ & $\begin{array}{c}-148.052011 \\
-2.3630 \\
-1.3008\end{array}$ & $\begin{array}{l}1.307 \\
1.524\end{array}$ & 23977 & $\begin{array}{c}-148.045169 \\
-2.3632 \\
-1.2998\end{array}$ & $\begin{array}{l}1.307 \\
1.524\end{array}$ & 22580 & $\begin{array}{l}-2.3687 \\
-1.3012\end{array}$ & $\begin{array}{l}1.305 \\
1.524\end{array}$ & 22577 \\
\hline
\end{tabular}


TABLE IV. (Continued.)

\begin{tabular}{|c|c|c|c|c|c|c|c|c|c|}
\hline & \multicolumn{3}{|c|}{ CG-AIMP } & \multicolumn{3}{|c|}{$\begin{array}{c}\text { Spin-orbit corrected } \\
\text { CG-AIMP }\end{array}$} & \multicolumn{3}{|c|}{$\begin{array}{c}\text { All-electron } \\
\text { numerical CG-HF }\end{array}$} \\
\hline & $\begin{array}{l}-0.8078 \\
-0.2197 \\
-0.2043\end{array}$ & $\begin{array}{l}0.785 \\
2.658 \\
4.184\end{array}$ & $\begin{array}{r}2189 \\
938\end{array}$ & $\begin{array}{l}-0.8081 \\
-0.2197 \\
-0.2043\end{array}$ & $\begin{array}{l}0.785 \\
2.658 \\
4.184\end{array}$ & $\begin{array}{r}2193 \\
943\end{array}$ & $\begin{array}{l}-0.8063 \\
-0.2196 \\
-0.2047\end{array}$ & $\begin{array}{l}0.785 \\
2.659 \\
4.179\end{array}$ & $\begin{array}{r}2193 \\
943\end{array}$ \\
\hline Ho $\left(f^{10} d^{1} s^{2}\right)^{6} L$ & $\begin{array}{c}-169.410279 \\
-2.4246 \\
-1.3272 \\
-0.8092 \\
-0.2180 \\
-0.2070\end{array}$ & $\begin{array}{l}1.283 \\
1.499 \\
0.766 \\
2.642 \\
4.133\end{array}$ & $\begin{array}{r}25710 \\
2421 \\
984\end{array}$ & $\begin{array}{c}-169.402026 \\
-2.4249 \\
-1.3261 \\
-0.8098 \\
-0.2181 \\
-0.2070\end{array}$ & $\begin{array}{l}1.283 \\
1.499 \\
0.766 \\
2.642 \\
4.133\end{array}$ & $\begin{array}{r}24100 \\
2425 \\
981\end{array}$ & $\begin{array}{l}-2.4322 \\
-1.3274 \\
-0.8076 \\
-0.2178 \\
-0.2075\end{array}$ & $\begin{array}{l}1.281 \\
1.499 \\
0.766 \\
2.644 \\
4.127\end{array}$ & $\begin{array}{r}24097 \\
2425 \\
981\end{array}$ \\
\hline $\operatorname{Er}\left(f^{11} d^{1} s^{2}\right)^{5} L$ & $\begin{array}{c}-192.722449 \\
-2.4906 \\
-1.3528 \\
-0.8069 \\
-0.2176 \\
-0.2098\end{array}$ & $\begin{array}{l}1.259 \\
1.475 \\
0.749 \\
2.620 \\
4.082\end{array}$ & $\begin{array}{r}27539 \\
2669 \\
1039\end{array}$ & $\begin{array}{c}-192.712555 \\
-2.4906 \\
-1.3512 \\
-0.8070 \\
-0.2176 \\
-0.2098\end{array}$ & $\begin{array}{l}1.259 \\
1.475 \\
0.749 \\
2.620 \\
4.082\end{array}$ & $\begin{array}{r}26695 \\
2673 \\
1029\end{array}$ & $\begin{array}{l}-2.4962 \\
-1.3533 \\
-0.8058 \\
-0.2175 \\
-0.2102\end{array}$ & $\begin{array}{l}1.257 \\
1.475 \\
0.749 \\
2.622 \\
4.078\end{array}$ & $\begin{array}{r}25691 \\
2673 \\
1029\end{array}$ \\
\hline $\operatorname{Tm}\left(f^{12} d^{1} s^{2}\right)^{4} K$ & $\begin{array}{c}-218.043452 \\
-2.5548 \\
-1.3794 \\
-0.8029 \\
-0.2153 \\
-0.2125\end{array}$ & $\begin{array}{l}1.237 \\
1.452 \\
0.733 \\
2.608 \\
4.034\end{array}$ & $\begin{array}{r}29474 \\
2933 \\
1086\end{array}$ & $\begin{array}{c}-218.031781 \\
-2.5548 \\
-1.3775 \\
-0.8030 \\
-0.2153 \\
-0.2125\end{array}$ & $\begin{array}{l}1.237 \\
1.452 \\
0.733 \\
2.608 \\
4.034\end{array}$ & $\begin{array}{r}27373 \\
2937 \\
1068\end{array}$ & $\begin{array}{l}-2.5623 \\
-1.3798 \\
-0.8022 \\
-0.2153 \\
-0.2131\end{array}$ & $\begin{array}{l}1.235 \\
1.451 \\
0.733 \\
2.610 \\
4.027\end{array}$ & $\begin{array}{r}27370 \\
2937 \\
1068\end{array}$ \\
\hline $\mathrm{Yb} \quad\left(f^{13} d^{1} s^{2}\right)^{3} H$ & $\begin{array}{c}-245.522441 \\
-2.6227 \\
-1.4082 \\
-0.8071 \\
-0.2069 \\
-0.2160\end{array}$ & $\begin{array}{l}1.217 \\
1.429 \\
0.717 \\
2.634 \\
3.982\end{array}$ & $\begin{array}{r}31529 \\
3217 \\
1091\end{array}$ & $\begin{array}{c}-245.508535 \\
-2.6227 \\
-1.4059 \\
-0.8072 \\
-0.2068 \\
-0.2160\end{array}$ & $\begin{array}{l}1.217 \\
1.429 \\
0.717 \\
2.634 \\
3.982\end{array}$ & $\begin{array}{r}29128 \\
3220 \\
1073\end{array}$ & $\begin{array}{l}-2.6309 \\
-1.4079 \\
-0.8052 \\
-0.2069 \\
-0.2168\end{array}$ & $\begin{array}{l}1.213 \\
1.429 \\
0.717 \\
2.636 \\
3.972\end{array}$ & $\begin{array}{r}29125 \\
3220 \\
1073\end{array}$ \\
\hline $\operatorname{Lu} \quad\left(f^{14} d^{1} s^{2}\right)^{2} D$ & $\begin{array}{c}-275.264284 \\
-2.6940 \\
-1.4397 \\
-0.8197 \\
-0.1878 \\
-0.2215\end{array}$ & $\begin{array}{l}1.195 \\
1.407 \\
0.701 \\
2.738 \\
3.912\end{array}$ & $\begin{array}{r}33687 \\
3520 \\
1014\end{array}$ & $\begin{array}{c}-275.247996 \\
-2.6941 \\
-1.4370 \\
-0.8198 \\
-0.1878 \\
-0.2215\end{array}$ & $\begin{array}{l}1.195 \\
1.407 \\
0.701 \\
2.738 \\
3.912\end{array}$ & $\begin{array}{r}30971 \\
3525 \\
1005\end{array}$ & $\begin{array}{l}-2.7050 \\
-1.4399 \\
-0.8189 \\
-0.1877 \\
-0.2223\end{array}$ & $\begin{array}{l}1.192 \\
1.407 \\
0.702 \\
2.743 \\
3.904\end{array}$ & $\begin{array}{r}30968 \\
3525 \\
1005\end{array}$ \\
\hline
\end{tabular}

whose parameters $\left\{B_{k}^{n l, I}, \beta_{k}^{n l, I}\right\}$ are determined through weighted least-squares fitting to the radial part of the WoodBoring spin-orbit operator ${ }^{6}$

$$
V_{\mathrm{SO}, n l}(r)=\frac{\alpha^{2}}{\left(2+\alpha^{2}\left[\epsilon_{n l}-V(r)\right]\right) r} \frac{d V(r)}{d r} .
$$

Herein, $\alpha$ is the fine-structure constant, $\epsilon_{n l}$ are the orbital energies of the spin-free relativistic equations of Cowan and Griffin, and $V(r)$ is an $X \alpha$ approximation to the HartreeFock one-electron potential. ${ }^{5}$ This effective one-electron spin-orbit operator includes an average of two-electron contributions through the use of the $X \alpha$ Hartree-Fock (HF) potential $V(r)$, although its detailed relationship to a meanfield spin-orbit operator is unknown. Also, an atomic scaling factor $\lambda^{I}$ is included in Eq. (8) which was first empirically parametrized $^{7}$ and later found to be unnecessary; ${ }^{12}$ consequently, we use $\lambda^{I}=1$. The $\left\{B_{k}, \beta_{k}\right\}$ parameters for the lanthanide elements and for the actinide elements have been produced here and they are presented in Tables II and III, respectively.

\section{B. Spin-free-state-shifted WB-AIMP Hamiltonian}

The spin-free relativistic $H^{\text {CG-AIMP }}$ Hamiltonian [Eq. (2)] is used in standard nonrelativistic methods. The spindependent $H^{\text {WB-AIMP }}$ Hamiltonian [Eq. (1)] is used in spinorbit configuration interaction (CI) calculations, i.g., in a basis of double-group symmetry-adapted determinantal functions with $\mathrm{HF}$ or complete active space self-consistent field (CASSCF) orbitals produced with the spin-free $H^{\text {CG-AIMP }}$ Hamiltonian. In order to treat electron correlation and spin-orbit interactions simultaneously at the highest possible level, the spin-free-state-shifted ( $s f s s)$ Hamiltonian $H_{s f s s}^{\text {WB-AIMP }}$ was introduced ${ }^{13}$

$$
\begin{aligned}
H_{s f s s}^{\mathrm{WB}-\mathrm{AIMP}}= & H^{\mathrm{WB}-\mathrm{AIMP}}+\sum_{i S M_{S} \Gamma \gamma} \delta(i S \Gamma)\left|\Phi^{\mathcal{P}}\left(i S M_{S} \Gamma \gamma\right)\right\rangle \\
& \times\left\langle\Phi^{\mathcal{P}}\left(i S M_{S} \Gamma \gamma\right)\right|
\end{aligned}
$$

with

$\delta(i S \Gamma)=\left[E^{\mathcal{G}}(i S \Gamma)-E^{\mathcal{G}}(\right.$ G.S. $\left.)\right]-\left[E^{\mathcal{P}}(i S \Gamma)-E^{\mathcal{P}}(\right.$ G.S. $\left.)\right]$. 
TABLE V. Spin-free relativistic valence energy, orbital energies, radial expectation values (in atomic units), and orbital spin-orbit coupling constants $\zeta_{n l}$ (in $\mathrm{cm}^{-1}$ ). The $\zeta_{n l}$ are calculated with the Gaussian orbitals and the analytical operators of Table III in the CG-AIMP calculations, and with the numerical orbitals and operators [Eq. (10)] in the all-electron numerical Cowan-Griffin-Hartree-Fock calculations. The AIMP calculations correspond to a $[\mathrm{Xe}, 4 f, 5 d]$ core and a $6 s, 6 p, 5 f, 6 d, 7 s$ valence.

\begin{tabular}{|c|c|c|c|c|c|c|c|c|c|}
\hline & \multicolumn{3}{|c|}{ CG-AIMP } & \multicolumn{3}{|c|}{$\begin{array}{l}\text { Spin-orbit corrected } \\
\text { CG-AIMP }\end{array}$} & \multicolumn{3}{|c|}{$\begin{array}{c}\text { All-electron } \\
\text { numerical CG-HF }\end{array}$} \\
\hline & $\begin{array}{l}\mathrm{E}(\mathrm{val}) \\
\epsilon(6 s) \\
\epsilon(6 p) \\
\epsilon(5 f) \\
\epsilon(6 d) \\
\epsilon(7 s)\end{array}$ & $\begin{array}{l}\langle r\rangle_{6 s} \\
\langle r\rangle_{6 p} \\
\langle r\rangle_{5 f} \\
\langle r\rangle_{6 d} \\
\langle r\rangle_{7 s}\end{array}$ & $\begin{array}{l}\zeta_{6 p} \\
\zeta_{5 f} \\
\zeta_{6 d}\end{array}$ & $\begin{array}{l}\mathrm{E}(\mathrm{val}) \\
\epsilon(6 s) \\
\epsilon(6 p) \\
\epsilon(5 f) \\
\epsilon(6 d) \\
\epsilon(7 s)\end{array}$ & $\begin{array}{l}\langle r\rangle_{6 s} \\
\langle r\rangle_{6 p} \\
\langle r\rangle_{5 f} \\
\langle r\rangle_{5 d} \\
\langle r\rangle_{7 s}\end{array}$ & $\begin{array}{l}\zeta_{6 p} \\
\zeta_{5 f} \\
\zeta_{6 d}\end{array}$ & $\begin{array}{l}\epsilon(6 s) \\
\epsilon(6 p) \\
\epsilon(5 f) \\
\epsilon(6 d) \\
\epsilon(7 s)\end{array}$ & $\begin{array}{l}\langle r\rangle_{6 s} \\
\langle r\rangle_{6 p} \\
\langle r\rangle_{5 f} \\
\langle r\rangle_{6 d} \\
\langle r\rangle_{7 s}\end{array}$ & $\begin{array}{l}\zeta_{6 p} \\
\zeta_{5 f} \\
\zeta_{6 d}\end{array}$ \\
\hline Th $\quad\left(5 f^{1} 6 d^{2} 7 s^{1}\right)^{5} I$ & $\begin{array}{l}-35.551931 \\
-1.9148 \\
-0.9673 \\
-0.1842 \\
-0.1901 \\
-0.2050\end{array}$ & $\begin{array}{l}1.580 \\
1.917 \\
1.904 \\
3.400 \\
4.484\end{array}$ & $\begin{array}{r}39264 \\
1113 \\
1243\end{array}$ & $\begin{array}{l}-35.473682 \\
-1.9149 \\
-0.9543 \\
-0.1842 \\
-0.1901 \\
-0.2050\end{array}$ & $\begin{array}{l}1.580 \\
1.917 \\
1.094 \\
3.400 \\
4.484\end{array}$ & $\begin{array}{r}31776 \\
1107 \\
1276\end{array}$ & $\begin{array}{l}-1.9144 \\
-0.9655 \\
-0.1849 \\
-0.1906 \\
-0.2050\end{array}$ & $\begin{array}{l}1.581 \\
1.919 \\
1.905 \\
3.394 \\
4.481\end{array}$ & $\begin{array}{r}31761 \\
1107 \\
1276\end{array}$ \\
\hline $\mathrm{Pa} \quad\left(5 f^{3} 6 d^{1} 7 s^{1}\right)^{6} L$ & $\begin{array}{c}-42.719219 \\
-1.8981 \\
-0.9216 \\
-0.1650 \\
-0.1737 \\
-0.1839\end{array}$ & $\begin{array}{l}1.556 \\
1.900 \\
1.766 \\
3.476 \\
4.689\end{array}$ & $\begin{array}{r}41342 \\
1345 \\
1199\end{array}$ & $\begin{array}{c}-42.630610 \\
-1.8981 \\
-0.9070 \\
-0.1651 \\
-0.1729 \\
-0.1839\end{array}$ & $\begin{array}{l}1.556 \\
1.900 \\
1.766 \\
3.476 \\
4.689\end{array}$ & $\begin{array}{r}33155 \\
1344 \\
1337\end{array}$ & $\begin{array}{l}-1.8990 \\
-0.9198 \\
-0.1660 \\
-0.1740 \\
-0.1842\end{array}$ & $\begin{array}{l}1.555 \\
1.903 \\
1.769 \\
3.471 \\
4.686\end{array}$ & $\begin{array}{r}33136 \\
1344 \\
1337\end{array}$ \\
\hline $\mathrm{U} \quad\left(5 f^{3} 6 d^{1} 7 s^{2}\right)^{5} K$ & $\begin{array}{l}-51.057518 \\
-2.1292 \\
-1.0767 \\
-0.3869 \\
-0.2250 \\
-0.1971\end{array}$ & $\begin{array}{l}1.499 \\
1.820 \\
1.440 \\
3.003 \\
4.392\end{array}$ & $\begin{array}{r}47440 \\
1907 \\
1810\end{array}$ & $\begin{array}{l}-50.950588 \\
-2.1293 \\
-1.0590 \\
-0.3870 \\
-0.2248 \\
-0.1971\end{array}$ & $\begin{array}{l}1.499 \\
1.820 \\
1.440 \\
3.003 \\
4.392\end{array}$ & $\begin{array}{r}37726 \\
1899 \\
1882\end{array}$ & $\begin{array}{l}-2.1313 \\
-1.0739 \\
-0.3881 \\
-0.2254 \\
-0.1973\end{array}$ & $\begin{array}{l}1.497 \\
1.823 \\
1.439 \\
2.996 \\
4.389\end{array}$ & $\begin{array}{r}37696 \\
1899 \\
1882\end{array}$ \\
\hline Np $\quad\left(5 f^{4} 6 d^{1} 7 s^{2}\right)^{6} L$ & $\begin{array}{l}-60.452972 \\
-2.2083 \\
-1.1077 \\
-0.4403 \\
-0.2336 \\
-0.1993\end{array}$ & $\begin{array}{l}1.465 \\
1.781 \\
1.362 \\
2.912 \\
4.335\end{array}$ & $\begin{array}{r}51521 \\
2216 \\
2001\end{array}$ & $\begin{array}{l}-60.329895 \\
-2.2084 \\
-1.0873 \\
-0.4404 \\
-0.2333 \\
-0.1993\end{array}$ & $\begin{array}{l}1.465 \\
1.782 \\
1.362 \\
2.912 \\
4.335\end{array}$ & $\begin{array}{r}40607 \\
2207 \\
2099\end{array}$ & $\begin{array}{l}-2.2122 \\
-1.1041 \\
-0.4413 \\
-0.2338 \\
-0.1997\end{array}$ & $\begin{array}{l}1.462 \\
1.785 \\
1.361 \\
2.907 \\
4.330\end{array}$ & $\begin{array}{r}40574 \\
2207 \\
2099\end{array}$ \\
\hline $\mathrm{Pu} \quad\left(5 f^{5} 6 d^{1} 7 s^{2}\right)^{7} K$ & $\begin{array}{l}-71.016672 \\
-2.2867 \\
-1.1375 \\
-0.4835 \\
-0.2356 \\
-0.2020\end{array}$ & $\begin{array}{l}1.433 \\
1.746 \\
1.297 \\
2.862 \\
4.276\end{array}$ & $\begin{array}{r}55790 \\
2538 \\
2159\end{array}$ & $\begin{array}{l}-70.875130 \\
-2.2868 \\
-1.1140 \\
-0.4836 \\
-0.2355 \\
-0.2020\end{array}$ & $\begin{array}{l}1.433 \\
1.746 \\
1.297 \\
2.862 \\
4.276\end{array}$ & $\begin{array}{r}43560 \\
2527 \\
2223\end{array}$ & $\begin{array}{l}-2.2926 \\
-1.1332 \\
-0.4845 \\
-0.2358 \\
-0.2026\end{array}$ & $\begin{array}{l}1.429 \\
1.749 \\
1.297 \\
2.857 \\
4.269\end{array}$ & $\begin{array}{r}43515 \\
2527 \\
2223\end{array}$ \\
\hline Am $\quad\left(5 f^{7} 6 d^{1} 7 s^{1}\right)^{10} D$ & $\begin{array}{c}-82.954076 \\
-2.1823 \\
-1.0210 \\
-0.3420 \\
-0.1386 \\
-0.1987\end{array}$ & $\begin{array}{l}1.425 \\
1.752 \\
1.305 \\
3.636 \\
4.405\end{array}$ & $\begin{array}{r}56865 \\
2671 \\
1295\end{array}$ & $\begin{array}{l}-82.800880 \\
-2.1824 \\
-0.9955 \\
-0.3422 \\
-0.1385 \\
-0.1987\end{array}$ & $\begin{array}{l}1.425 \\
1.752 \\
1.305 \\
3.636 \\
4.405\end{array}$ & $\begin{array}{r}43907 \\
2666 \\
1332\end{array}$ & $\begin{array}{l}-2.1916 \\
-1.0167 \\
-0.3434 \\
-0.1391 \\
-0.1993\end{array}$ & $\begin{array}{l}1.417 \\
1.756 \\
1.305 \\
3.631 \\
4.399\end{array}$ & $\begin{array}{r}43901 \\
2666 \\
1332\end{array}$ \\
\hline $\mathrm{Cm} \quad\left(5 f^{7} 6 d^{1} 7 s^{2}\right)^{9} D$ & $\begin{array}{l}-96.003066 \\
-2.4420 \\
-1.1958 \\
-0.5894 \\
-0.2110 \\
-0.2097\end{array}$ & $\begin{array}{l}1.375 \\
1.682 \\
1.190 \\
2.904 \\
4.148\end{array}$ & $\begin{array}{r}64846 \\
3232 \\
2216\end{array}$ & $\begin{array}{c}-95.818048 \\
-2.4421 \\
-1.1650 \\
-0.5896 \\
-0.2110 \\
-0.2097\end{array}$ & $\begin{array}{l}1.375 \\
1.682 \\
1.190 \\
2.904 \\
4.148\end{array}$ & $\begin{array}{r}49660 \\
3221 \\
2216\end{array}$ & $\begin{array}{l}-2.4546 \\
-1.1896 \\
-0.5900 \\
-0.2109 \\
-0.2107\end{array}$ & $\begin{array}{l}1.369 \\
1.686 \\
1.189 \\
2.902 \\
4.124\end{array}$ & $\begin{array}{r}49587 \\
3221 \\
2216\end{array}$ \\
\hline Bk $\quad\left(5 f^{8} 6 d^{1} 7 s^{2}\right)^{8} H$ & $\begin{array}{c}-110.295651 \\
-2.5310 \\
-1.2326 \\
-0.5997 \\
-0.1908 \\
-0.2154\end{array}$ & $\begin{array}{l}1.347 \\
1.649 \\
1.153 \\
2.993 \\
4.071\end{array}$ & $\begin{array}{r}70023 \\
3587 \\
2113\end{array}$ & $\begin{array}{c}-110.083976 \\
-2.5312 \\
-1.1975 \\
-0.5999 \\
-0.1908 \\
-0.2154\end{array}$ & $\begin{array}{l}1.347 \\
1.649 \\
1.154 \\
2.993 \\
4.071\end{array}$ & $\begin{array}{r}53099 \\
3563 \\
2092\end{array}$ & $\begin{array}{l}-2.5483 \\
-1.2252 \\
-0.5999 \\
-0.1903 \\
-0.2168\end{array}$ & $\begin{array}{l}1.339 \\
1.654 \\
1.153 \\
2.993 \\
4.052\end{array}$ & $\begin{array}{r}53012 \\
3564 \\
2092\end{array}$ \\
\hline $\mathrm{Cf} \quad\left(5 f^{9} 6 d^{1} 7 s^{2}\right)^{7} K$ & $\begin{array}{c}-126.050792 \\
-2.6149 \\
-1.2640\end{array}$ & $\begin{array}{l}1.321 \\
1.620\end{array}$ & 75379 & $\begin{array}{c}-125.808218 \\
-2.6150 \\
-1.2239\end{array}$ & $\begin{array}{l}1.321 \\
1.620\end{array}$ & 56566 & $\begin{array}{l}-2.6370 \\
-1.2554\end{array}$ & $\begin{array}{l}1.312 \\
1.625\end{array}$ & 56459 \\
\hline
\end{tabular}


TABLE V. (Continued.)

\begin{tabular}{|c|c|c|c|c|c|c|c|c|c|}
\hline & & AIMP & & Spin- & $\begin{array}{l}t \text { correct } \\
\text { AIMP }\end{array}$ & & & $\begin{array}{l}\text {-electro } \\
\text { ical CC }\end{array}$ & \\
\hline & $\begin{array}{l}-0.6216 \\
-0.1834 \\
-0.2195\end{array}$ & $\begin{array}{l}1.119 \\
3.016 \\
4.009\end{array}$ & $\begin{array}{l}3957 \\
2134\end{array}$ & $\begin{array}{l}-0.6218 \\
-0.1834 \\
-0.2195\end{array}$ & $\begin{array}{l}1.119 \\
3.016 \\
4.009\end{array}$ & $\begin{array}{l}3927 \\
2084\end{array}$ & $\begin{array}{l}-0.6212 \\
-0.1826 \\
-0.2214\end{array}$ & $\begin{array}{l}1.119 \\
3.018 \\
3.986\end{array}$ & $\begin{array}{l}3927 \\
2084\end{array}$ \\
\hline Es $\left(5 f^{10} 6 d^{1} 7 s^{2}\right)^{6} L$ & $\begin{array}{c}-143.299786 \\
-2.6934 \\
-1.2921 \\
-0.6484 \\
-0.1819 \\
-0.2228\end{array}$ & $\begin{array}{l}1.296 \\
1.593 \\
1.087 \\
3.000 \\
3.957\end{array}$ & $\begin{array}{r}80937 \\
4348 \\
2220\end{array}$ & $\begin{array}{c}-143.024278 \\
-2.6936 \\
-1.2466 \\
-0.6485 \\
-0.1818 \\
-0.2228\end{array}$ & $\begin{array}{l}1.296 \\
1.593 \\
1.087 \\
3.000 \\
3.957\end{array}$ & $\begin{array}{r}60000 \\
4312 \\
2137\end{array}$ & $\begin{array}{l}-2.7234 \\
-1.2820 \\
-0.6474 \\
-0.1808 \\
-0.2251\end{array}$ & $\begin{array}{l}1.285 \\
1.598 \\
1.087 \\
3.005 \\
3.929\end{array}$ & $\begin{array}{r}59966 \\
4312 \\
2136\end{array}$ \\
\hline Fm $\quad\left(5 f^{11} 6 d^{1} 7 s^{2}\right)^{5} L$ & $\begin{array}{c}-162.091586 \\
-2.7881 \\
-1.3174 \\
-0.6703 \\
-0.1811 \\
-0.2269\end{array}$ & $\begin{array}{l}1.269 \\
1.567 \\
1.057 \\
2.981 \\
3.895\end{array}$ & $\begin{array}{r}86744 \\
4753 \\
2314\end{array}$ & $\begin{array}{c}-161.780053 \\
-2.7883 \\
-1.2659 \\
-0.6705 \\
-0.1809 \\
-0.2269\end{array}$ & $\begin{array}{l}1.269 \\
1.567 \\
1.057 \\
2.981 \\
3.895\end{array}$ & $\begin{array}{r}63631 \\
4714 \\
2210\end{array}$ & $\begin{array}{l}-2.8107 \\
-1.3075 \\
-0.6712 \\
-0.1806 \\
-0.2287\end{array}$ & $\begin{array}{l}1.260 \\
1.573 \\
1.057 \\
2.981 \\
3.874\end{array}$ & $\begin{array}{r}63593 \\
4714 \\
2210\end{array}$ \\
\hline Md $\quad\left(5 f^{12} 6 d^{1} 7 s^{2}\right)^{4} K$ & $\begin{array}{c}-182.428815 \\
-2.8791 \\
-1.3437 \\
-0.6921 \\
-0.1785 \\
-0.2310\end{array}$ & $\begin{array}{l}1.244 \\
1.542 \\
1.030 \\
2.974 \\
3.837\end{array}$ & $\begin{array}{r}92894 \\
5180 \\
2387\end{array}$ & $\begin{array}{c}-182.076753 \\
-2.8794 \\
-1.2856 \\
-0.6923 \\
-0.1783 \\
-0.2310\end{array}$ & $\begin{array}{l}1.244 \\
1.542 \\
1.030 \\
2.975 \\
3.837\end{array}$ & $\begin{array}{r}67406 \\
5135 \\
2258\end{array}$ & $\begin{array}{l}-2.9007 \\
-1.3333 \\
-0.6937 \\
-0.1784 \\
-0.2326\end{array}$ & $\begin{array}{l}1.235 \\
1.548 \\
1.030 \\
2.974 \\
3.819\end{array}$ & $\begin{array}{r}67365 \\
5135 \\
2258\end{array}$ \\
\hline No $\left(5 f^{13} 6 d^{1} 7 s^{2}\right)^{3} H$ & $\begin{array}{c}-204.427555 \\
-2.9759 \\
-1.3716 \\
-0.7203 \\
-0.1689 \\
-0.2365\end{array}$ & $\begin{array}{l}1.219 \\
1.518 \\
1.004 \\
3.029 \\
3.772\end{array}$ & $\begin{array}{r}99397 \\
5629 \\
2344\end{array}$ & $\begin{array}{c}-204.029919 \\
-2.9762 \\
-1.3059 \\
-0.7206 \\
-0.1686 \\
-0.2365\end{array}$ & $\begin{array}{l}1.219 \\
1.519 \\
1.004 \\
3.029 \\
3.772\end{array}$ & $\begin{array}{r}71318 \\
5581 \\
2212\end{array}$ & $\begin{array}{l}-2.9945 \\
-1.3604 \\
-0.7226 \\
-0.1695 \\
-0.2379\end{array}$ & $\begin{array}{l}1.212 \\
1.525 \\
1.003 \\
3.022 \\
3.757\end{array}$ & $\begin{array}{r}71272 \\
5581 \\
2212\end{array}$ \\
\hline $\operatorname{Lr} \quad\left(5 f^{14} 6 d^{1} 7 s^{2}\right)^{2} D$ & $\begin{array}{c}-228.159302 \\
-3.0797 \\
-1.4042 \\
-0.7595 \\
-0.1488 \\
-0.2453\end{array}$ & $\begin{array}{l}1.196 \\
1.495 \\
0.978 \\
3.210 \\
3.692\end{array}$ & $\begin{array}{r}106326 \\
6103 \\
2093\end{array}$ & $\begin{array}{c}-227.709948 \\
-3.0801 \\
-1.3300 \\
-0.7599 \\
-0.1485 \\
-0.2453\end{array}$ & $\begin{array}{l}1.196 \\
1.495 \\
0.978 \\
3.210 \\
3.692\end{array}$ & $\begin{array}{r}75399 \\
6053 \\
1967\end{array}$ & $\begin{array}{l}-3.0965 \\
-1.3926 \\
-0.7630 \\
-0.1492 \\
-0.2467\end{array}$ & $\begin{array}{l}1.189 \\
1.502 \\
0.978 \\
3.207 \\
3.679\end{array}$ & $\begin{array}{r}75349 \\
6054 \\
1967\end{array}$ \\
\hline
\end{tabular}

Here, $\mathcal{P}$ and $\mathcal{G}$ are two CI spaces of, respectively, small and large relative size; $\mathcal{P}$ is required to be good enough for the calculation of the spin-orbit couplings but not for the electron correlation effects, for which the much larger $\mathcal{G}$ space is necessary. $\Phi^{\mathcal{P}}\left(i S M_{S} \Gamma \gamma\right)$ are spin-free CI wave functions in the small space and G.S. refers to the ground state, but it can be any given spin-free state. This $s f s s$-Hamiltonian is a practical means to take advantage of the fact that electron correlation is handled with a much larger efficiency with spin-free Hamiltonians than with spin-dependent Hamiltonians. Its use is based on the assumption that correlation and spin orbit can be decoupled to a large extent. A sfss-spin-orbit calculation requires performing correlated spin-free calculations with the $H^{\text {CG-AIMP }}$ Hamiltonian using the $\mathcal{G}$ and $\mathcal{P}$ spaces and one final spin-orbit CI calculation with the $H_{s f s s}^{\text {WB-AIMP }}$ Hamiltonian using the $\mathcal{P}$ space. A $\mathcal{P}$ space made of the significant reference configurations plus single excitations which can partially take care of spin-orbit polarizations has been proven to be very efficient in $\mathrm{Ir}^{+},{ }^{12} \mathrm{Pt},{ }^{11}$ and $\mathrm{Ce} .{ }^{14}$

\section{Atomic valence basis sets}

Once the relativistic core $a b$ initio model potentials have been obtained as summarized in Sec. II A, what remains is the production of the valence basis sets. In order to do so, the $H^{\text {CG-AIMP }}$ Hamiltonian [Eq. (2)] is used in atomic valenceonly Hartree-Fock calculations and the exponents and coefficients of Gaussian atomic orbitals are optimized by minimization of the valence SCF energy using standard allelectron methods. ${ }^{32}$ After this, every single $p, d$, and $f$ valence atomic orbital is spin-orbit corrected: ${ }^{7}$ The coefficient of the innermost Gaussian primitive is changed and the orbital is renormalized, in such a way that the orbital spinorbit coupling constants calculated with the numerical Cowan-Griffin-Hartree-Fock atomic orbital and with the analytical spin-orbit-corrected valence orbital coincide. This procedure has been shown to significantly improve the spinorbit dependent properties at a time so that the quality of the bonding related properties is maintained. ${ }^{7}$

Following this procedure, we obtained spin-orbitcorrected relativistic valence basis sets for the lanthanide elements $\mathrm{Ce}-\mathrm{Lu}$ and for the actinide elements Th-Lr. They are presented in the Supplementary Material Section of Ref. 26 as E-PAPS document file. The radial functions of the valence orbitals of $\mathrm{Sm}$ and $\mathrm{Pu}$ are presented in Figs. 1 and 2; their similarity to the all-electron numerical orbitals is clear. The atomic valence properties that correspond to these basis 
TABLE VI. Spectroscopic constants for the ${ }^{1} \Sigma^{+}$ground state of ThO.

\begin{tabular}{lccc}
\hline \hline & $R_{e} / \AA$ & $\omega_{e} / \mathrm{cm}^{-1}$ & $D_{e} / \mathrm{eV}$ \\
\hline Experiment $^{\mathrm{a}}$ & 1.840 & 896 & 9.0 \\
SCF calculations & & & \\
Küechle $e t$ al. $^{\mathrm{b}}$ & 1.829 & 943 & 6.07 \\
This work $^{\mathrm{c}}$ & 1.819 & 956 & 5.99 \\
CASSCF calculations $^{(8}$ electrons in & orbitals) & \\
Küechle $e t$ al. $^{\mathrm{b}}$ & 1.882 & 876 & 8.92 \\
This work $^{\mathrm{c}}$ & 1.886 & 865 & 9.15 \\
\hline \hline
\end{tabular}

${ }^{\mathrm{a}}$ Reference 35

${ }^{\mathrm{b}}$ Reference 17. Relativistic energy-adjusted pseudopotential calculation corresponding to a $[\mathrm{Kr}, 4 d, 4 f]$ core and a $(12 s 11 p 10 d 8 f) /[8 s 7 p 6 d 4 f]$ basis set.

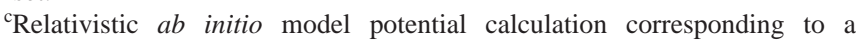
$[\mathrm{Xe}, 4 f, 5 d]$ core and a $(14 s 10 p 11 d 9 f) /[6 s 5 p 5 d 4 f]$ basis set.

sets are shown in Tables IV and V, where it is clear that they are very similar to the corresponding all-electron calculations. The basis sets are minimal valence basis sets of the size shown in Table I. When they are used in molecular calculations, their flexibility can be enhanced by adding or releasing the outermost primitives and by extension with appropriate functions, such as polarization and diffuse functions. For lanthanide elements, very stable results with respect to the basis set size are found when a number of outermost primitives are added to the minimal basis set up to a number of $(14 s 10 p 9 d 8 f) /[6 s 5 p 5 d 4 f]$ basis set functions (see Ref. 14). The errors due to the use of smaller [ $4 s 3 p 3 d 3 f]$ basis sets were $0.015 \AA$ in bond distance, 10 $\mathrm{cm}^{-1}$ in vibrational frequency, and $0.2 \mathrm{eV}$ in dissociation energy. ${ }^{14}$ For actinide elements, a good performance is obtained as well with the same basis set size. The good quality of the results on the ${ }^{1} \Sigma^{+}$ground state of ThO using a $(14 s 10 p 11 d 9 f) /[6 s 5 p 5 d 4 f]$ basis set for Th and the same basis set for $\mathrm{O}$ as in Ref. 14, is shown in Table VI. Although several calculations on ThO exist, ${ }^{33,34}$ we compare our results with those of Ref. 17, which use correct choices of basis set and of core/valence partition. Our results are essentially coincident with those of the energy-adjusted pseudopotential (EAPP) calculations with a slightly smaller core, ${ }^{17}$ as was the case of transition metals, ${ }^{11}$ and the comparison with experimental data is good having in mind that dynamical correlation effects are missing. (These effects have been previously found to be very similar in EAPP and AIMP calculations. ${ }^{11}$ )

\section{CONCLUSIONS}

Starting from atomic Cowan-Griffin calculations, we produced and presented here the ingredients of the WBAIMP method for the lanthanide elements $\mathrm{Ce}$ to $\mathrm{Lu}$ and for the actinide elements $\mathrm{Th}$ to $\mathrm{Lr}$, what completes the chemically relevant part of the Periodic Table. These are: relativistic $[\mathrm{Kr}, 4 d]$ core AIMPs and Wood-Boring spin-orbit operators for $\mathrm{Ce}-\mathrm{Lu}$, together with optimized minimal $(14 s 10 p 9 d 8 f) /[2 s 1 p 1 d 1 f]$ Gaussian valence basis sets, and relativistic $[\mathrm{Xe}, 4 f, 5 d]$ core AIMPs and Wood-Boring spin-orbit operators for Th-Lr, together with optimized minimal $(14 s 10 p 11 d 9 f) /[2 s 1 p 1 d 1 f]$ Gaussian valence basis sets. A [6s5p5d4f] contraction is recommended for all these 28 elements in molecular calculations. The atomic and molecular results show the same good quality already observed for the main-group elements and the transition metal elements.

\section{ACKNOWLEDGMENT}

This work was partly supported by a grant from Ministerio de Educación y Cultura, Spain (No. DGESIC, PB980108).

${ }^{1}$ J. C. Phillips and L. Kleinman, Phys. Rev. 116, 287 (1959).

${ }^{2}$ S. Huzinaga and A. A. Cantu, J. Chem. Phys. 55, 5543 (1971).

${ }^{3}$ L. Seijo and Z. Barandiarán, in Computational Chemistry: Reviews of Current Trends, edited by J. Leszczynski (World Scientific, Singapore, 1999), Vol. 4, p. 55.

${ }^{4}$ S. Huzinaga, L. Seijo, Z. Barandiarán, and M. Klobukowski, J. Chem. Phys. 86, 2132 (1987).

${ }^{5}$ R. D. Cowan and D. C. Griffin, J. Opt. Soc. Am. 66, 1010 (1976).

${ }^{6}$ J. H. Wood and A. M. Boring, Phys. Rev. B 18, 2701 (1978).

${ }^{7}$ L. Seijo, J. Chem. Phys. 102, 8078 (1995).

${ }^{8}$ L. Seijo, Z. Barandiarán, and S. Huzinaga, J. Chem. Phys. 91, 7011 (1989).

${ }^{9}$ Z. Barandiarán, L. Seijo, and S. Huzinaga, J. Chem. Phys. 93, 5843 (1990).

${ }^{10}$ Z. Barandiarán and L. Seijo, Can. J. Chem. 70, 409 (1992).

${ }^{11}$ M. Casarrubios and L. Seijo, J. Chem. Phys. 110, 784 (1999).

${ }^{12}$ F. Rakowitz, M. Casarrubios, L. Seijo, and C. M. Marian, J. Chem. Phys. 108, 7980 (1998).

${ }^{13}$ R. Llusar, M. Casarrubios, Z. Barandiarán, and L. Seijo, J. Chem. Phys. 105, 5321 (1996).

${ }^{14}$ S. Díaz-Megías and L. Seijo, Chem. Phys. Lett. 299, 613 (1999).

${ }^{15}$ M. Dolg, H. Stoll, and H. Preuss, J. Mol. Struct.: THEOCHEM 231, 243 (1991).

${ }^{16}$ M. Dolg, H. Stoll, and H. Preuss, J. Chem. Phys. 90, 1730 (1989).

${ }^{17}$ W. Küchle, M. Dolg, H. Stoll, and H. Preuss, J. Chem. Phys. 100, 7535 (1994).

${ }^{18}$ R. B. Ross, S. Gayen, and W. C. Ermler, J. Chem. Phys. 100, 8145 (1994).

${ }^{19}$ W. C. Ermler, R. B. Ross, and P. A. Christiansen, Int. J. Quantum Chem. 40, 829 (1991).

${ }^{20}$ T. R. Cundari and W. J. Stevens, J. Chem. Phys. 98, 5555 (1993).

${ }^{21}$ M. Douglas and N. M. Kroll, Ann. Phys. (N.Y.) 82, 89 (1974).

${ }^{22}$ B. A. Hess, Phys. Rev. A 33, 3742 (1986).

${ }^{23}$ F. Rakowitz, C. M. Marian, L. Seijo, and U. Wahlgren, J. Chem. Phys. 110, 3678 (1999).

${ }^{24}$ F. Rakowitz, C. M. Marian, and L. Seijo, J. Chem. Phys. 111, 10436 (1999).

${ }^{25}$ Detailed core AIMP and valence basis set data libraries in electronic format are available from the authors upon request or directly at the address http://www.qui.uam.es/Data/AIMPLibs.html. See also Ref. 29.

${ }^{26}$ See AIP Document No. E-JCPSA6-114-311102 for AIMP parameters and valence basis sets. This document may be retrieved via the E-PAPS homepage (http://www.aip.org/pubservs/epaps.html) or from ftp.aip.org in the directory /epaps/. See the EPAPS homepage for more information.

${ }^{27}$ S. Katsuki and S. Huzinaga, Chem. Phys. Lett. 147, 597 (1988).

${ }^{28}$ E. Francisco, L. Seijo, and L. Pueyo, J. Solid State Chem. 63, 391 (1986).

${ }^{29}$ MOLCAS version 4, K. Andersson, M. R. A. Blomberg, M. P. Fülscher et al., Lund University, Sweden, 1997.

${ }^{30}$ C. C. J. Roothaan, Rev. Mod. Phys. 32, 179 (1960).

${ }^{31}$ R. M. Pitzer and N. W. Winter, J. Phys. Chem. 92, 3061 (1988).

${ }^{32}$ Gaussian Basis Sets for Molecular Calculations, edited by S. Huzinaga (Elsevier, Amsterdam, 1984).

${ }^{33}$ C. M. Marian, U. Wahlgren, O. Gropen, and P. Pyykkö, J. Mol. Struct.: THEOCHEM 169, 339 (1988).

${ }^{34}$ G. L. Malli, R. Salahub, and M. Zerner, ACS Symp. Ser. 394, 291 (1989).

${ }^{35}$ K. P. Huber and G. Herzberg, Molecular Spectra and Molecular Structure of Diatomic Molecules (Van Nostrand, New York, 1979). 